\title{
Autophagic Flux Impairment Exacerbates Myocardial Ischemia-Reperfusion Injury in Experimental Diabetes Through Atg5/LAMP2 Cleavage by Calpains
}

\section{Lichun Guan}

ShangHai General Hospital

\section{Ziqin Yu}

Shanghai Institute of Cardiovascular Diseases, Zhongshan hospital

\section{Zhimei Che}

South Campus, Renji Hospital

\section{Hang Zhang}

Nanjing First Hospital

\section{Yong Yu}

Shanghai Zhongshan Hospital

\section{Dicheng Yang}

Shanghai General Hospital

\section{Ruizhen Chen}

Shanghai Zhongshan Hospital

Min Yu ( $\nabla$ minyudr@163.com)

ShangHai General Hospital https://orcid.org/0000-0002-0042-2196

\section{Original investigation}

Keywords: Diabetes, Myocardial ischemia-reperfusion (I/R) injury, Autophagic flux, Calpain, Atg5, LAMP2

Posted Date: August 4th, 2020

DOI: https://doi.org/10.21203/rs.3.rs-42392/v1

License: @ (i) This work is licensed under a Creative Commons Attribution 4.0 International License. Read Full License 


\section{Abstract}

Background: is a chronic metabolic disease characterized by hyperglycemia, which has negative effects on cardiac revascularization in patients with coronary artery disease (CAD).

Methods: This study focused on the role of autophagic flux in regulating susceptibility of STZ-induced diabetic heart to ischemia-reperfusion (I/R) injury. We established STZ-induced diabetes mice and perform I/R process by coronary artery ligation, and neonatal mice cardiomyocytes culture subjected to high glucose and hypoxia/reoxygenation(H/R).

Results: Diabetic heart was more sensitive to I/R injury. Autophagic flux, represented by TEM, relative LC3 $₫$ changes under CQ intervention and P62 expression, was impaired in diabetic hearts and deteriorated during subsequent I/R. Calpains were activated in diabetic I/R heart, and the inhibition of calpains partially rescued autophagic flux, cardiac function, and cell death. The expression of autophagic flux related proteins Atg5 and LAMP2 decreased significantly in diabetic l/R heart. Further studies suggested that calpain could cleave Atg5 and LAMP2, and generate cleaved fragments, which might be reversed by calpain inhibition. Inhibition of calpain囚or in company with overexpression of Atg5 and LAMP2, could reduce myocardial injury in diabetic heart subject to I/R. But overexpression of Atg5 alone could worsen the I/R injury.

Conclusion: In conclusion, calpain-mediated cleavage of Atg5 and LAMP2 accounts for the impaired autophagic flux which increases the susceptibility to myocardial I/R injury in experimental diabetic mice.

\section{Background}

Although revascularization is an important therapeutic measure to improve cardiac function and prognosis in patients with coronary heart disease(CAD), myocardial reperfusion therapy itself can also cause myocardial injury, known as myocardial ischemia-reperfusion injury (MIRI)[1]. Diabetes is a global rapidly growing metabolic disease, which damages cardiac function and increases susceptibility to MIRI. Clinical studies have shown that the CAD patients with diabetes, especially insulin dependent patients, had worse cardiac outcomes after revascularization[2]. The underlying mechanisms by which diabetes sensitizes the myocardium to MIRI remain to be fully elucidated.

Autophagy is a highly conserved process of degradation of self-proteins, such as long-lived cytoplasmic proteins and damaged organelles, to maintain the stability of intracellular environment $[3,4]$. Autophagy activity is measured by autophagic flux, which is defined as a dynamic process of autophagy, from the formation of autophagosomes to the clearance of autophagosomes. The mount of LC3II in the presence and absence of late autophagic flux inhibitors, such as chloroquine (CQ), can be used to detect autophagy flux; if autophagy flux is occurring, the LC3II will be higher in the presence of the CQ[5]. The SQSTM1/P62 protein serves as a link between LC3 and ubiquitinated substrates. In addition, SQSTM1/P62-bound polyubiquitinated proteins become incorporated into the completed autophagosome and are degraded in autolysosomes. Therefore, SQSTM1/p62 can also be used as 
autophagy flux marker, at least in some settings[5]. It has been reported that autophagic flux is impaired during MIRI, and the recovery of autophagic flux will reduce the oxidative stress injury of cardiomyocytes and alleviate MIRI[6-8]. Suppressed autophagic process is also found in experimental diabetic cardiomyopathy, while upregulating autophagy restores myocardial function $(10,11)$. However, there is few studies on the role of autophagic flux in MIRI under the background of diabetes.

Calpains belongs to the calcium-dependent thiol-protease family, fifteen gene products of the calpain family have been identified to date. Among them, $\mu$-calpain (calpain-1) and m-calpain (calpain-2) were the main isoforms of calpains expressed in cardiomyocytes[9]. Calpastatin, which tightly regulates Calpain-1 and calpain-2, is an endogenous inhibitor that explicitly inhibits calpains, but not other cysteine proteases. Over-expression of calpastatin has been extensively used to inhibit calpain in a variety of in vitro and in vivo models[10,11]. Calpains are involved in autophagic process by cleavage of numerous autophagy-related proteins, including Atg5[12]. Atg5 is an essential protein during the formation of autophagic vesicles. It is activated and then forms a complex with ATG12, which is necessary for LC3-I conjugation to PE (phosphatidylethanolamine) to form LC3-II[13]. Calpain1/2 could cleave ATG5 into $24 \mathrm{KD}$ fragments, therefore block the formation of autophagosome in various cell types [14-16]. Calpains have been discovered to be one of the main components regulating autophagy in the process of I/R injury of retinal cells and hepatocytes through cleavage of Atg7 and Becline1[17, 18]. Our former research demonstrated calpain1/2 would be activated under cardiomyocytes I/R stress, suppress mitochondrial fusion and contribute to MIRI[19]. However, the role of calpains in autophagic flux during MIRI and its substrates has not been reported.

Lysosomes play a crucial role in the clearance of autophagosomes, and lysosome-associated membrane protein 2 (LAMP2) is a kind of single-span lysosome membrane protein, which maintains lysosome stability and participates in autophagic flux [20]. LAMP2 deficient mice have impaired autophagic flux in several tissues[21, 22]. In neuronal cells, the cleavage of LAMP2 could contribute to neuronal death due to the impairment of the autophagic flux and lysosomal damage mediated by calpain activation[23]. Similarly, no studies have reported whether activated calpains would cleave LMAP2 in cardiomyocytes.

In this study, we employed Streptozotocin (STZ)-induced diabetic mice and transgenic calpastatinoverexpression mouse to be challenged with myocardial I/R, and primary cardiomyocytes to be treated with high glucose $(H G)$ and hypoxia/reoxygenation $(H / R)$. we investigated the role of autophagic flux in diabetes related sensitivity to MIRI in vivo and in vivo, and clarified calpain-mediated regulation on autophagic flux through degradation of Atg5 and LAMP2.

\section{Methods}

\section{Animals}

Male inbred C57BL/6 mice aging 6-8 weeks were used for the experiment. The transgenic calpastatinoverexpression mouse strain (Tg-CAST) was provided by the laboratory of Tianqing PENG (Lawson Health Research Institute, Canada)[24] and was bred in the Department of Laboratory Animal Science, 
Fudan University in an SPF environment. All animal experiments were approved by the Ethics Review Board for Animal Studies of Shanghai Jiao Tong University School of Medicine (approval no. SYKX-20080050; Shanghai, China) and were conducted in strict accordance with the Guide for the Care and Use of Laboratory Animals published by the US National Institutes of Health (NIH Publication, 8th Edition, 2011).

\section{Induction of Diabetes and Experimental Protocol}

Diabetes was induced in overnight fasted mice by administering a single intraperitoneal (i.p.) injection of $50 \mathrm{mg} / \mathrm{kg}$ streptozotocin (STZ) freshly dissolved in $0.1 \mathrm{~mol} / \mathrm{L}$ sodium citrate buffer (pH 4.5). The mice in the control group were injected with a similar volume $(100 \mu \mathrm{l})$ of sodium citrate buffer alone. The mice whose fasting blood glucose level was more than $16.7 \mathrm{mmol} / \mathrm{L}$ after five days of injection were diabetic (Supplementary material Table 1). All mice were fed for one month. Furthermore, Diabetic cardiomyopathy mice were excluded by cardiac ultrasound, and diabetic mice with normal heart function (the indexes of myocardial function have no obvious difference with the sham group) were randomized to different groups.

\section{Identification of transgenic mice}

All Tg-CAST littermates were genotyped by DNA electrophoresis following the protocol of PENG's lab as previously described[19].

\section{Ischemia/reperfusion model establishment}

Animals were randomly divided into sham operation groups and I/R operation groups for the following interventions. Mice were anesthetized with $2 \%$ isoflurane inhalation with an isoflurane delivery system (Viking Medical, Medford, NJ) without ventilation. Then we made a $1 \mathrm{~cm}$ incision in the left fourth intercostal space. The left coronary artery (LCA) was ligated (no ligation for sham operation groups) for $30 \mathrm{~min}$ followed by $12 \mathrm{~h}$ of reperfusion as previously described[19]. At the indicated time points, the mice were sacrificed by cervical dislocation, and the hearts were immediately extracted for further analysis.

\section{Myocardial infarct size measurement}

Myocardial infarct size was measured using Evans blue (Sigma囚USA, 17779هE2129) and 2, 3, 5-triphenyl tetrazolium chloride (TTC) staining as previously described[19].

\section{Echocardiography}

Echocardiography was conducted by M-mode echocardiography using a Philips IE33 instrument (Philips Medical Systems Corporation, Andover, MA, USA) with a 1-5 MHz transducer (S5-1), under the instruction of the guideline. Two-dimensional guided M-mode measurements of the LV internal diameter were obtained from the short-axis view at the level of the papillary muscles over at least three beats and were averaged. Computer algorithms were used to count left ventricular ejection fraction (LVEF), LV end- 
systolic dimension (LVESD), left ventricular fraction shortening (LVFS), and LV end-diastolic dimension (LVEDD) (Supplementary material table 2).

\section{Serum cTnl detection}

Serum cTnl level was detected by an Elisa kit (ab188877, Abcam) following the manufacturer's instructions to evaluate myocardial I/R injury.

\section{Cardiomyocyte culture and induction of Hypoxia/reoxygenation (H/R) based on high- glucose (HG).}

Neonatal mouse (1 days old, Department of Laboratory Animal Science, Fudan University) ventricular cardiomyocytes were isolated as previously described with a few modifications[19]. In brief, the hearts of neonatal mice were isolated surgically, and the ventricles were enzymatically digested in $0.08 \%$ collagenase II for $8 \mathrm{~min}$ in an automatic heat regulator at $37^{\circ} \mathrm{C}$. After centrifugation and resuspension, cells were preplaced for $2 \mathrm{~h}$ in Dulbecco's-modified Eagle's medium (DMEM) supplemented with $10 \%$ fetal bovine serum (Gibco) to reduce fibroblasts, then plated in culture dishes and incubated at $37{ }^{\circ} \mathrm{C}$ in a $5 \%$ $\mathrm{CO} 2$ incubator. DMEM with glucose concentration of $5.5 \mathrm{mmol} / \mathrm{L}$ was taken as the control group (NG) and $33 \mathrm{mmol} / \mathrm{L}$ as the $\mathrm{HG}$ group, and the cells were treated for $48 \mathrm{~h}$. Then, to mimic the $\mathrm{I} / \mathrm{R}$ injury, myocytes were treated with hypoxia/reoxygenation(H/R). Briefly, the isolated myocardial cells, adhered to the well with normal pulsation, were moved to a hypoxia incubator ( $1 \% 02,94 \% \mathrm{~N} 2$ and $5 \% \mathrm{CO} 2)$ for $12 \mathrm{~h}$, followed by reoxygenation in normoxic conditions for $3 \mathrm{~h}$ at $37^{\circ} \mathrm{C}$.

\section{Cardiomyocyte viability assay}

Cultured cardiomyocyte viability was assessed by Cell Counting Kit-8 (C0038, Beyotime Biotechnology, China) as previously described[19].

\section{Cell death measurement}

Cardiomyocyte death was measured by annexin V-FITC. The Annexin V-FITC Apoptosis Detection Kit microscopy (C1062, Beyotime Biotechnology, China) was applied under the manufacturer's instructions for apoptosis detection. In the early stages of apoptosis, cells were Annexin V-positive, while cells that were both PI- and Annexin V-positive were at the later stages whereas cells that were only PI-positive were necrotic.

To localize and evaluate quantitatively cells undergoing apoptosis in the cardiomyocytes, in situ terminal deoxynucleotidyl transferase-mediated dUTP nick-end labelling (TUNEL) was performed using an in-situ apoptosis detection kit (C1098, Beyotime Biotechnology, China). We carried out our experiment in accordance with the instructions.

\section{Western blot analysis}

The area of I/R injury in the heart was collected in an EP tube with RIPA protein lysis buffer containing protease inhibitors. Protein concentrations were measured with a BCA assay kit (P0010, Beyotime 
Biotechnology, China). The protein obtained above was separated by $10-15 \%$ SDS-PAGE and then transferred onto a polyvinylidene difluoride (PVDF, Millipore, USA) membrane, placed in 5\% BSA containing TBST at room temperature for $1 \mathrm{~h}$ and then incubated overnight at $4^{\circ} \mathrm{C}$ with primary antibody diluted in TBST with 5\% BSA. Primary antibodies include: LC3 (1:1000, 4108, CST), SQSTM1/P62 (1:1000, 23214, CST), calpain1(1:1000, 2556, CST), calpain2(1:1000, 2539, CST), Beclin-1 (1:1000, 3495, CST), ATG5(1:1000, 2630, CST), LAMP2(1:1000, ab13524, Abcam). The PVDF membranes were washed and then incubated with HRP-conjugated secondary antibodies at room temperature for $1 \mathrm{~h}$. GAPDH $(1: 10,000,5174, C S T)$ were used as the internal control. An ECL-HRP chemiluminescence kit (34577, Thermo Fisher, USA) was used to visualize the bands which were quantified by Image Lab Software (BioRad, USA). All samples were analyzed in triplicate with a multilabel reader (excitation, $360 \mathrm{~nm}$; emission, $460 \mathrm{~nm}$, Thermo, America).

\section{IHC staining}

Heart tissues were embedded in paraffin and cut into 10 $\mu \mathrm{m}$ slices. Each slice was deparaffinized using xylene and an ethanol gradient. Antigen retrieval was performed by boiling in $10 \mathrm{mM}$ citrate buffer for 10 min and cooling for 30 min. Endogenous peroxidase activity was quenched with $3 \% \mathrm{H} 2 \mathrm{O} 2$ for 10 min, and the slices then were washed three times in PBS for $2 \mathrm{~min} .1 \%$ goat serum was used to block nonspecific binding. The slices were incubated at $4{ }^{\circ} \mathrm{C}$ overnight with a $1: 50$ dilution of anti-LC3II or SQSTM1/P62 antibody, and then were washed two times in PBS for $5 \mathrm{~min}$. The samples were incubated with a biotinylated goat anti-rabbit secondary antibody (1:200, ab150077, Abcam) for $45 \mathrm{~min}$. The samples were added tri-antibody (SAB complex) for $40 \mathrm{~min}$. The samples were observed by DAB chromogenic method, and certain brown strength was taken as positive.

\section{Measurement of LDH level}

The cell culture medium was collected after various treatments to measure the LDH levels using an LDH kit (C0016, Beyotime Biotechnology, China;) according to the manufacturer's instructions.

\section{Calpain activity detection}

A fluorometric calpain activity assay kit ((ab65308, Abcam) was used to quantify calpain activity. In simple terms, the lysates of myocardial cells or heart tissue were centrifuged, and the supernatant was used to detect calpain activity using the fluorescent substrate N-succinyl-LLVY-AMC. All samples were analyzed in triplicate with a multilabel reader (excitation, $360 \mathrm{~nm}$; emission, $460 \mathrm{~nm}$, Thermo, America) and expressed as relative fluorescent units (RFU).

\section{Quantitative real-time reverse transcription PCR (qRT-PCR)}

Total RNAs were extracted from primary cardiomyocytes using Trizol Reagent (Invitrogen, USA; 15596018). 500 ng RNA per sample was used for reverse transcription and qRT-PCR assay. The qRT-PCRs were performed using SYBR Premix Ex Taq (TaKaRa, Japan; Cat. No. RR420A) according to the manufacturer's instructions on an ABI Prism 7500 Real-Time PCR System (Applied Biosystems, Foster 
City, CA, USA). $\beta$-actin was used as internal control. The data were analyzed by using comparative CT method. The primers used in this study for qRT-PCR were as follows:

$\beta$-actin

Forward: 5'-AGAGCCTCGCCTTTGCCGAT-3'

Reverse: 5'- TGCCAGATTTTCTCCATGTCGT -3'

ATG5

Forward: 5'-GGACCTTCTACACTGTCCATCC-3'

Reverse: 5'-TGTCATTCTGCAGTCCCATC-3'

LAMP2

Forward: 5'-CTAGGAGCCGTTCAGTCCAA-3'

Reverse: 5'-CTTGCAGGTGAATACCCCAA-3'

\section{Adenoviral infection}

In order to overexpress ATG5 and LAMP2, Ad-vector, Ad-ATG5 and Ad-LAMP2 were synthesized by HanBio, China. Cardiomyocytes were infected with each of the three adenoviruses. An empty adenovirus (Ad-vector) served as a control at a multiplicity of infection of $100 \mathrm{PFU} / c e l l$. The specific methods are as follows: cells were plated in DMEM with $10 \%$ fetal bovine serum at a density of $5 * 10^{5} / \mathrm{ml}$. 48 hours after plating, serum was removed and the cells were infected with recombinant adenovirus. 6 hours later, the culture medium containing $10 \%$ serum was added and incubated another $48-72$ hours cells. Successful transfection was verified by WB-analysis (Supplementary 1 A, B). For in vivo studies, adenoviruses are expressed in the heart by myocardial in situ injection at a titer of $4 \times 1010$ PFU/ mice. Exposing the mouse heart (the method is same as the establishment of I/R model), $10 \mu \mathrm{l}$ viral suspension were injected into the myocardium at four points around the heart's coronary arteries by using a microsyringe. After 3 days, mice were subjected to I/R (Supplementary1 D, E).

\section{Statistical analysis}

All statistical tests were analyzed with GraphPad Prism 6.02. All data were expressed as means \pm SD. Statistical comparisons were conducted using Student's t-test (for comparisons between two groups) or a one-way analysis of variance (ANOVA, for comparisons of three or more groups) followed by the Bonferroni post-hoc test. $\mathrm{P}<0.05$ was considered statistically significant.

\section{Results}




\section{Autophagic flux correlates with I/R injury of STZ-induced diabetic mice}

We first evaluate the difference of the survival rate between the wild group and the diabetic group with the extension of reperfusion time (Fig1 A). To further investigate the correlation of autophagy flux with diabetic MIRI, we tested the expression of autophagic flux markers LC3II and SQSTM1/P62 by immunohistochemical (IHC) staining in heart samples of diabetic and wild-type (WT) mice subjected 12h reperfusion. The STZ+I/R group hearts expressed significantly lower levels of LC3II, but higher levels of SQSTM1/P62 (Fig1 B, C). In the two I/R group, we evaluated the correlation between P62 and EF value. Interestingly, we found the expression of SQSTM1/P62 was inversely correlated with the LVEF value $\left(R^{2}=0.8292, P<0.05\right)(F i g 1 \mathrm{D})$. These results indicate that reduced LC3II and increased P62 are associated with diabetic myocardial I/R injury.

\section{Experimental diabetic myocytes are more susceptible to I/R injury in vivo and in vitro.}

The STZ-induced mice were used as a diabetic model and wild-type mice were used as controls in this study. The heart tissue was obtained at $30 \mathrm{~min}$ of ischemia and $12 \mathrm{~h}$ of reperfusion. The extent of infract size was minimal and similar between diabetic mice and wild-type mice (This is consistent with our selection). I/R induced myocardial infarction in both groups, and the diabetic mice showed larger infarction ratio than WT mice (Fig2 A, B). The serum cTnl, a marker of myocardial injury, was less than 1 $\mathrm{ng} / \mathrm{ml}$ in either of the sham groups. After I/R, diabetic mice showed higher serum cTnl than wild-type (Fig2 C). Functionally, we used echocardiography to analyze LVEF in mice. The results showed that LVEF value of STZ mice in sham group decreased slightly compared with WT group, but there was no statistical significance. I/R reduced the heart function significantly, and LVEF of diabetic mice dropped to lower extend than WT mice (Fig2 D, E). Other indexes including FS, LVEDD and LVESD was shown in supplement1 (table2). In vitro, cell viability was decreased under HG condition, while apoptosis rose compared with the NG control. Under H/R stress, HG-conditioned myocytes appeared less cell viability and more apoptosis than NG myocytes. (Fig2F-H).

\section{Impaired autophagic flux on diabetes during IR injury}

To investigate the effects of diabetes on cardiomyocytes autophagic flux during $\mathrm{I} / \mathrm{R}$, we first need to observe the formation of autophagosomes. In the ultrastructure, transmission electron microscopy (TEM) showed that a certain amount of autophagosome were formed in WT mice accompanied by neat muscle fibers and intact dense mitochondria. However, the formation of autophagosome in STZ mice was minimal as well as a few mitochondrial vacuoles were observed. I/R injury accelerated the formation of autophagosome, and mitochondria became vacuolated with disordered crista. Nonetheless, STZ mice after I/R surgery showed a decrease in autophagic lysosomes, but more serious mitochondrial vacuoles and damaged crista (Fig3A). In accordance with TEM, Western-blot revealed that LC3II and Beclin1 were downregulated in STZ mice (Fig3B, C), but upregulated in I/R mice (Fig3D, E). Compared to wild type I/R mice, the two protein showed some decline in STZ+l/R mice (Fig3 D, E). 
As mentioned above, autophagy flux can be evaluated by comparing the expression of LC3II with and without CQ intervention, or by combining the expression of LC3II and SQSTM1/P62. WB results showed the relative expressions of LC3II in the four groups under CQ administration respectively. In WT sham group, we defined the ratio of LC3II expression of mice with CQ intraperitoneally injection and those without injection as basal autophagic flux. The results revealed that the autophagic flux were impaired after STZ or I/R condition ( $2.8 \pm 0.2$ basically, $1.56 \pm 0.15$ in $\mathrm{I} / \mathrm{R}, 1.54 \pm 0.12$ in STZ), and STZ+l/R group showed the lowest autophagic flux (1.09 \pm 0.19$)$ (Fig3 F). In addition, we also measured SQSTM1/P62 levels in four groups respectively. Sham mice in WT had lower SQSTM1/P62 abundance, higher abundance of SQSTM1/P62, which indicated impaired autophagic flux, were detected in I/R and in STZ group. Highest SQSTM1/P62 was found in STZ+1/R group which was in agreement with the results of LC3II expression under CQ intervention (Fig3G, H). We further observed LC3Il level intuitively through immunofluorescence in cultured cardiomyocytes, and the results were consistent with WB results in vivo (Fig3 I, J).

\section{Regulating autophagic flux impact I/R injury in the diabetic heart}

To investigate the influence of autophagic flux on diabetic heart with I/R injury, we treated the STZ mice with rapamycin (Rapa, $1 \mathrm{mg} / \mathrm{kg}$ ), an inhibitor of $\mathrm{mTOR}$, to motivate autophagic flux, and 3-methyladenine (3-MA, $30 \mathrm{mg} / \mathrm{kg}$ ), an inhibitor of type III phosphatidylinositol 3-kinases (PI-3K), to suppress autophagic flux, or CQ (50 mg/kg), respectively. Rapamycin significantly decreased the areas of myocardial infraction and serum cTnl levels after I/R stress, compared with NS controls (Equal volume saline treatment), while 3-MA and CQ both elevated the infarction area and serum CTnI (Fig4 A-C). In cardiomyocyte H/R experiments, Flow cytometry apoptosis assay also showed reduced cell apoptosis in the rapamycin $(0.1 \mu \mathrm{M})$ treatment group under $\mathrm{H} / \mathrm{R}$ and $\mathrm{HG}$ condition. Conversely, autophagic flux inhibitor 3-MA (5mM) and $\mathrm{CQ}$ resulted in aggravating apoptosis in $\mathrm{H} / \mathrm{R}+\mathrm{HG}$ myocytes. These results dedicate that autophagic flux is involved in regulating I/R injury of diabetic heart (Fig4 D, E).

\section{Calpain 1,2 activation aggravates $\mathrm{I} / \mathrm{R}$ injury in diabetic hearts}

To investigate the potential involvement of calpains in autophagic flux impairment, calpains expression and activity were determined. Immunohistochemical and WB showed higher expression of calpain 1 but not calpain 2 in the diabetic heart. calpain 1 and calpain 2 were all up-regulated in $1 / R$ and STZ+I/R mice (Fig5 A-D). Meanwhile, calpain activity also increased, especially in STZ+I/R mice (Fig5 E). Calpastatin inhibits calpain's activity with no effect on calpain expression, so we used CAST transgenic mice to testify the impact of calpain on autophagic flux. This results have been verified in our previous study[19]. The results of Evans-blue TTC and echocardiography showed that CAST mice could resist the attack of diabetes and I/R on myocardium, exhibiting a smaller myocardial infarction area and a higher LVEF value (Fig5 F-I). Autophagic flux under STZ+I/R was detected in WT and CAST by LC3II expression changes under CQ intervention. Relative LC3II expression was recovered in CAST either in STZ treatment $(2.41 \pm 0.26$ vs $1.63 \pm 0.12)$ or in STZ+l/R stress $(2.34 \pm 0.33$ vs $1.17 \pm 0.11)$. (Fig5 J). CAST also attenuated P62 accumulation after STZ+I/R stress compared to the WT (Fig5 K, L). These data indicated calpain 
inhibition restore autophagic flux. PD150606 is an inhibitor of calpain activity. PD150606 effectively inhibited calpain activation induced by $\mathrm{H} / \mathrm{R}$ and/or HG in cultured mice cardiomyocytes (Fig5 M), as well as apoptosis detected by TUNEL assay (Fig $5 \mathrm{~N}, 0$ ). We also detected LC3II expression by immunofluorescence which showed autophagic flux regained and agreed with those in vivo (Fig5 P, Q).

\section{Cleavage of LAMP2 and ATG5 by calpains during I/R in experimental diabetic heart}

We then explored the underlying mechanism of reduced autophagic flux during I/R in diabetic heart. LAMP2 and ATG5 were highly expressed at the WT control mice, and both diabetes and I/R alone could result in LAMP2 and ATG5 reduction. I/R stress in diabetic heart further decreased the two proteins expression. Notably, LAMP2 decreased significantly in I/R heart than in the diabetic (Fig6 A, B). In order to understand the role of calpain in autophagic flux related proteins, we first verified the inhibition effect of CAST mice on calpain activity. Compared to diabetic $\mathrm{l} / \mathrm{R}$ heart, calpain protein expression and activity of WT and CAST heart were at low levels. Overexpressed calpastatin significantly inhibited calpain activation in diabetic I/R hearts but did not inhibit its expression. We study the protein expression of LAMP2 and ATG5 in CAST transgenic mice. When calpain activity was inhibited by calpastatin overexpression in STZ+1/R mice, LAMP2 and ATG5 expression were partially restored (Fig6 C-E). We also checked mRNA expression of LAMP2 and ATG5 by qRT-PCR. Surprisingly, there were no decrement of the two mRNA levels in diabetic hearts, and even increment in I/R models (Fig6 F). As LAMP2 and ATG5 were substrate of calpains, we supposed that the two autophagic flux proteins were cleaved by activated calpains in diabetic heart, I/R heart, and diabetic I/R heart respectively.

In vitro, we found calpain inhibitor PD150606 treatment could reverse the reduction of both LAMP2 and ATG5 in HG+H/R cardiomyocytes by immunofluorescence technique (Fig6 G). When LAMP2 and ATG5 were cleaved by calpains, $24 \mathrm{KD}$ cleaved ATG5 and 35KD cleaved LAMP2 could be detected. In vitro experiments showed the remarkable cleaved fragments of LAMP2 and ATG5 in HG+H/R cardiomyocytes, whereas inhibition of calpain by PD150606 hinder the cleavage (Fig6 H). Taken together, these results suggest that calpains activation contributes to LAMP2 and ATG5 depletion in diabetic heart with I/R injury.

\section{Overexpression of LAMP2 and ATG5 rescues autophagic flux but shows different effect on the diabetic I/R injury.}

To investigate the roles of LAMP2 and ATG5 in diabetic I/R injury, myocardium of STZ mice was injected with Ad-vector, Ad-LAMP2 and Ad-ATG5 in situ before I/R (supplymentary1 E). Evans-blue TTC staining showed transfection with ad-LAMP2 could reduce the size of myocardial infarction, although ad-ATG5 showed the opposite (Fig7 A, B). The possible reason is that tATG5, the fragment of ATG5 cleaved by calpain, can promote apoptosis[14]. So, overexpression of ATG5 would produce more tATG5 which cause more cellular loss. Then, we constructed the same models in transgenic mice and transfected the above three adenoviruses again. the results suggested that the myocardial infarction size of the mice in both overexpression groups was further reduced compared to the ad-vector group, and LAMP2 overexpression in CAST caused the least infarction area. (Fig7 A, B). 
In vitro experiment, we transfected all three types of adenoviruses in cardiomyocytes (supplymentary1 A, $B)$, and combined transfection with two adenoviruses carrying the target gene. Autophagic flux markers LC3II and P62 were detected by WB, ad-ATG5 and (or) ad-LAMP2 could partially increase LC3II and decrease P62, PD150606 administration could enhances such changes (Fig7 C, D). The restoration of autophagic flux was greatest when both ad-ATG5 and ad-LAMP2 were transfected simultaneously, followed by ad-LAMP2 alone (Fig7 C, D). Both CCK8 and LDH tests reflect the activity of cardiomyocytes, and their changes are consistent with in-vivo experiments. Overexpression of ATG5 suppressed cellular viability and deteriorated LDH release, while overexpression of LAMP2 protected cellular viability and mitigated LDH. With calpain inhibition, both LAMP2 and ATG5 overexpression further elevated cellular viability and blunted LDH release compared to ad-GFP transfection, and the combined transfection of adATG5 and ad-LAMP2 showed the greatest protective effect on HG+HR cardiomyocytes (Fig7 E, F). These results revealed how LAMP2 and ATG5 are involved in the impairment of autophagic flux and myocardial $\mathrm{l} / \mathrm{R}$ injury in diabetic mice.

\section{Discussion}

In this study, we illustrate that cardiomyocyte autophagic flux is impaired in myocardial diabetic I/R injury, contributing to cell apoptosis, increased infraction size and decreased EF value. Calpain $1 / 2$ upregulation and activation during diabetic $\mathrm{l} / \mathrm{R}$ is responsible for autophagic flux impairment. we found obvious diabetic l/R injury-induced degradation in ATG5 and LAMP2 which are the key proteins for autophagosome formation and autophagosome-lysosome fusion respectively and substrates of calpain1/2. Calpain cleavage of Atg5 and LAMP2 impairs autophagic flux. Inhibition of calpain activity,or overexpression of LAMP2, could restores autophagic flux and reduce myocardial injury in diabetic l/R. But overexpression of ATG5 alone appears to be deteriorating, possibly because tAtg5 is an apoptosis inducer.

Contemporary studies have demonstrated autophagy is attenuated in cardiomyocytes subjected to STZdiabetes[25], while enhanced to I/R stress[26, 27]. But autophagic flux was impaired in both diabetes and I/R condition, which is consisted with our results. We also demonstrated autophagic flux is impaired in diabetic hearts and deteriorated during I/R. The mechanism of diabetes-based myocardial injury is complex, while high glucose concentration can induce metabolic dysfunction and redox imbalance[28]. Adaptive autophagic flux in response to stress contribute to clear the damaging byproducts and exert a cytoprotective effect[6, 29-31]. The impairment of autophagic flux would result in excessive ROS, harmful protein fragments, and dysfunctional organelles, which will cause cell disruption and death[4]. In this study experimental diabetic l/R model revealed larger infarction ratio, higher cTnl release, lower heart function and survival, less cell viability and more apoptosis than WT I/R model. Furthermore, Rapamycin pretreatment to diabetic I/R mice could reduce myocardial injury by enhancing autophagic flux, but autophagic flux inhibitor 3-MA and CQ would worsen those injury. In a word, the impairment of autophagic flux occurred in experimental diabetic hearts and increased their sensitivity to I/R injury. 
Calpain activation has been reported to be involved in myocardial I/R injury though inducing ER stress, and in diabetic cardiomyopathy by cleavage of ATP5A1 and generating mitochondrial ROS[28, 32]. Our previous research also found calpain $1 / 2$ was activated during myocardial I/R process and destroyed mitochondrial dynamic balance[19]. It is worth to note that we found a markedly increment of calpain1 expression in diabetic hearts, but not calpain2. Meanwhile, calpain1 and calpain2 were increased in I/R heart. The expression and activity of calpains were the highest in diabetic heart with I/R. Some studies have revealed the relationship between calpain and autophagy. For instance, calpain 2-mediated cleavage of Atg3 and Atg7 inhibited autophagy in fatty livers, and worsen the I/R injury[17]. Calpain inhibitors would increase autophagosome and seem to be a therapeutic strategy for brain degeneration like Huntington's disease[33]. In this study, we also found inhibition of calpain by genetic overexpression of calpastatin or calpain inhibitor PD150606 would restore autophagic flux and attenuate I/R injury in diabetic heart or cardiomyocytes.

The occurrence of autophagy flux needs the participation of various autophagy-related proteins, in which ATG5 is essential in the formation of autophagosomes, and LAMP2 in the structure of autophagosomelysosome. The asynchronous expression of mRNA and protein indicated the enhancement of degradation of ATG5 and LAMP2 in diabetic I/R heart. These two proteins are substrates of calpain1/2. We detected the $24 \mathrm{~kb}$ ATG5 fragment and $35 \mathrm{~kb}$ LAMP2 fragment in HG + H/R stress cardiomyocytes, and these fragments could be diminished by calpain inhibition. These evidences confirmed the calpain cleavage of ATG5 and LAMP2.

To further clarify the role of ATG5 and LAMP2 in calpain-mediated autophagic flux impairment in diabetic I/R injury, we performed rescue experiment through overexpressing ATG5 and LAMP2 in cardiomyocytes by transfecting ad-ATG5 and ad-LAMP2. For the WT mice, LAMP2 overexpression could reduce heart injury, but ATG5 overexpression was not beneficial to I/R injury. We supposed such results might be due to the pro-apoptotic effect of tATG5 fragment [14]. However, in CAST mice both overexpression of ATG5 and LAMP2 could reduce myocardial injury. It seems that calpain inhibition counteracted the negative effects of ATG5 overexpression on myocardium. In vitro, we also gained the similar results as in vivo when we transfected ad-ATG5 and ad-LAMP2 to cultured cardiomyocytes and performed HG $+\mathrm{H} / \mathrm{R}$ stress. In addition, we checked the autophagic flux and found that overexpression of each of these two proteins could recover autophagic flux presented by LC3 $\triangle$ and P62. Such effect could be promoted when inhibiting calpain activity at the same time.

\section{Conclusion}

In conclusion, our study demonstrated that impaired autophagic flux is one of the key roles of increased sensitivity of diabetic heart to I/R injury. Calpain-mediated cleavage of ATG5 and LAMP2 accounts for the impaired autophagic flux. Targeting autophagic flux and calpain activity could be a promising strategy to improve heart function in diabetic patients with heart revascularization.

\section{Abbreviations}


Atg5: autophagy-related proteins

LAMP2: lysosome-associated membrane protein 2

I/R: Ischemia-reperfusion

Tg-CAST: Transgenic calpastatin-overexpression

STZ: Streptozotocin

LVEF: Left ventricular ejection fraction

LVESD: LV end-systolic dimension

LVFS: LV end-systolic dimension

LVEDD: LV end-diastolic dimension

H/R: Hypoxia/reoxygenation

HG: High- glucose

Rapa: Rapamycin

3-MA: 3-methyladenine

CQ: Chloroquine

\section{Declarations}

\section{Ethics approval and consent to participate}

All animal experiments were approved by the Ethics Review Board for Animal Studies of Shanghai Jiao Tong University School of Medicine (approval No. SYKX-2008-0050; Shanghai, China) and were conducted in strict accordance with the guide for the care and use of laboratory animals published by the US National Institutes of Health (NIH Publication, 8th Edition, 2011).

\section{Consent for publication}

All authors gave consent for publication

\section{Availability of data and materials}

The datasets used and/or analysed during the current study are available from the corresponding author on reasonable request. 
The authors declare that they have no conflicts of interest.

\section{Funding}

National Natural Science Foundation of China: 81770411, 81300094

\section{Authors' contributes}

The study was designed by Min Yu, Ruizhen Chen and Lichun Guan. The animal model was set up by Lichun Guan and Zhimei Che. The molecular biological experiment such as PCR, Wester blots, flowmetry study and immunofluorescence staining, were carried out by Lichun Guan, Ziqing Yu, Yong Yu. Cell culture and virus transfection were done by Lichun Guan. Morphological study such as TEM, EvansbluTTC staining, morphological examination was implemented by Lichun Guan. Finally, the statistical analysis and article writing were done by Min Yu, Lichun Guan, Dicheng Yang.

\section{Acknowledgements}

The transgenic calpastatin-overexpression mouse strain (Tg-CAST) was provided by the laboratory of Tianqing PENG (Lawson Health Research Institute, Canada)

\section{References}

1. Davidson SM, Ferdinandy P, Andreadou I, Botker HE, Heusch G, Ibanez B, Ovize M, Schulz R, Yellon DM, Hausenloy DJ et al: Multitarget Strategies to Reduce Myocardial Ischemia/Reperfusion Injury: JACC Review Topic of the Week. J Am Coll Cardio/ 2019, 73(1):89-99.

2. Reinstadler SJ, Stiermaier T, Eitel C, Metzler B, de Waha S, Fuernau G, Desch S, Thiele H, Eitel I: Relationship between diabetes and ischaemic injury among patients with revascularized ST-elevation myocardial infarction. Diabetes Obes Metab 2017, 19(12):1706-1713.

3. Komatsu M, Kurokawa H, Waguri S, Taguchi K, Kobayashi A, Ichimura Y, Sou YS, Ueno I, Sakamoto A, Tong KI et al: The selective autophagy substrate p62 activates the stress responsive transcription factor Nrf2 through inactivation of Keap1. Nat Cell Biol 2010, 12(3):213-223.

4. Levine B, Kroemer G: Autophagy in the pathogenesis of disease. Cell 2008, 132(1):27-42.

5. Klionsky DJ, Abdelmohsen K, Abe A, Abedin MJ, Abeliovich H, Acevedo Arozena A, Adachi H, Adams $\mathrm{CM}$, Adams PD, Adeli $\mathrm{K}$ et al: Guidelines for the use and interpretation of assays for monitoring autophagy (3rd edition). Autophagy 2016, 12(1):1-222.

6. Su Q, Liu Y, Lv XW, Ye ZL, Sun YH, Kong BH, Qin ZB: Inhibition of IncRNA TUG1 upregulates miR-142$3 p$ to ameliorate myocardial injury during ischemia and reperfusion via targeting HMGB1-and Rac1induced autophagy. Journal of molecular and cellular cardiology 2019, 133:12-25.

7. Wang JL, Li YZ, Tao TQ, Wang XR, Wang Y, Song DD, Liu XH: Postconditioning with Calreticulin Attenuates Myocardial Ischemia/Reperfusion Injury and Improves Autophagic Flux. Shock 2019. 
8. Qiao SG, Sun Y, Sun B, Wang A, Qiu J, Hong L, An JZ, Wang C, Zhang HL: Sevoflurane postconditioning protects against myocardial ischemia/reperfusion injury by restoring autophagic flux via an NO-dependent mechanism. Acta Pharmacol Sin 2019, 40(1):35-45.

9. Sorimachi $\mathrm{H}$, Ono Y: Regulation and physiological roles of the calpain system in muscular disorders. Cardiovasc Res 2012, 96(1):11-22.

10. Li X, Li Y, Shan L, Shen E, Chen R, Peng T: Over-expression of calpastatin inhibits calpain activation and attenuates myocardial dysfunction during endotoxaemia. Cardiovasc Res 2009, 83(1):72-79.

11. Galvez AS, Diwan A, Odley AM, Hahn HS, Osinska H, Melendez JG, Robbins J, Lynch RA, Marreez Y, Dorn GW, 2nd: Cardiomyocyte degeneration with calpain deficiency reveals a critical role in protein homeostasis. Circ Res 2007, 100(7):1071-1078.

12. Norman JM, Cohen GM, Bampton ET: The in vitro cleavage of the hAtg proteins by cell death proteases. Autophagy 2010, 6(8):1042-1056.

13. Otomo C, Metlagel Z, Takaesu G, Otomo T: Structure of the human ATG12 ATG5 conjugate required for LC3 lipidation in autophagy. Nature structural \& molecular biology 2013, 20(1):59-66.

14. Yousefi S, Perozzo R, Schmid I, Ziemiecki A, Schaffner T, Scapozza L, Brunner T, Simon HU: Calpainmediated cleavage of Atg5 switches autophagy to apoptosis. Nat Cell Bio/ 2006, 8(10):1124-1132.

15. Pyo JO, Jang MH, Kwon YK, Lee HJ, Jun JI, Woo HN, Cho DH, Choi B, Lee H, Kim JH et al: Essential roles of Atg5 and FADD in autophagic cell death: dissection of autophagic cell death into vacuole formation and cell death. J Biol Chem 2005, 280(21):20722-20729.

16. Mehrpour M, Esclatine A, Beau I, Codogno P: Overview of macroautophagy regulation in mammalian cells. Cell Res 2010, 20(7):748-762.

17. Zhao Q, Guo Z, Deng W, Fu S, Zhang C, Chen M, Ju W, Wang D, He X: Calpain 2-mediated autophagy defect increases susceptibility of fatty livers to ischemia-reperfusion injury. Cell Death Dis 2016, 7:e2186.

18. Russo R, Berliocchi L, Adornetto A, Varano GP, Cavaliere F, Nucci C, Rotiroti D, Morrone LA, Bagetta G, Corasaniti MT: Calpain-mediated cleavage of Beclin-1 and autophagy deregulation following retinal ischemic injury in vivo. Cell Death Dis 2011, 2:e144.

19. Guan L, Che Z, Meng X, Yu Y, Li M, Yu Z, Shi H, Yang D, Yu M: MCU Up-regulation contributes to myocardial ischemia-reperfusion Injury through calpain/OPA-1-mediated mitochondrial fusion/mitophagy Inhibition. J Cell Mol Med 2019, 23(11):7830-7843.

20. Eskelinen EL: Roles of LAMP-1 and LAMP-2 in lysosome biogenesis and autophagy. Mol Aspects Med 2006, 27(5-6):495-502.

21. Tanaka Y, Guhde G, Suter A, Eskelinen EL, Hartmann D, Lullmann-Rauch R, Janssen PM, Blanz J, von Figura K, Saftig P: Accumulation of autophagic vacuoles and cardiomyopathy in LAMP-2-deficient mice. Nature 2000, 406(6798):902-906.

22. Cuervo AM, Wong E: Chaperone-mediated autophagy: roles in disease and aging. Cell Res 2014, 24(1):92-104. 
23. Geronimo-Olvera C, Montiel T, Rincon-Heredia R, Castro-Obregon S, Massieu L: Autophagy fails to prevent glucose deprivation/glucose reintroduction-induced neuronal death due to calpain-mediated lysosomal dysfunction in cortical neurons. Cell Death Dis 2017, 8(6):e2911.

24. Li Y, Li Y, Feng Q, Arnold M, Peng T: Calpain activation contributes to hyperglycaemia-induced apoptosis in cardiomyocytes. Cardiovasc Res 2009, 84(1):100-110.

25. Xie Z, Lau K, Eby B, Lozano P, He C, Pennington B, Li H, Rathi S, Dong Y, Tian R et al: Improvement of cardiac functions by chronic metformin treatment is associated with enhanced cardiac autophagy in diabetic OVE26 mice. Diabetes 2011, 60(6):1770-1778.

26. Hamacher-Brady A, Brady NR, Gottlieb RA: Enhancing macroautophagy protects against ischemia/reperfusion injury in cardiac myocytes. J Biol Chem 2006, 281(40):29776-29787.

27. Ma X, Liu H, Foyil SR, Godar RJ, Weinheimer CJ, Hill JA, Diwan A: Impaired autophagosome clearance contributes to cardiomyocyte death in ischemia/reperfusion injury. Circulation 2012, 125(25):3170-3181.

28. Ni R, Zheng D, Xiong S, Hill DJ, Sun T, Gardiner RB, Fan GC, Lu Y, Abel ED, Greer PA et al: Mitochondrial Calpain-1 Disrupts ATP Synthase and Induces Superoxide Generation in Type 1 Diabetic Hearts: A Novel Mechanism Contributing to Diabetic Cardiomyopathy. Diabetes 2016, 65(1):255-268.

29. Kanamori H, Naruse G, Yoshida A, Minatoguchi S, Watanabe T, Kawaguchi T, Yamada Y, Mikami A, Kawasaki M, Takemura G et al: Metformin Enhances Autophagy and Provides Cardioprotection in delta-Sarcoglycan Deficiency-Induced Dilated Cardiomyopathy. Circ Heart Fail 2019, 12(4):e005418.

30. Yao Q, Ke ZQ, Guo S, Yang XS, Zhang FX, Liu XF, Chen X, Chen HG, Ke HY, Liu C: Curcumin protects against diabetic cardiomyopathy by promoting autophagy and alleviating apoptosis. Journal of molecular and cellular cardiology 2018, 124:26-34.

31. Xiao Y, Wu QQ, Duan MX, Liu C, Yuan Y, Yang Z, Liao HH, Fan D, Tang QZ: TAX1BP1 overexpression attenuates cardiac dysfunction and remodeling in STZ-induced diabetic cardiomyopathy in mice by regulating autophagy. Biochim Biophys Acta Mol Basis Dis 2018, 1864(5 Pt A):1728-1743.

32. Zheng D, Wang G, Li S, Fan GC, Peng T: Calpain-1 induces endoplasmic reticulum stress in promoting cardiomyocyte apoptosis following hypoxia/reoxygenation. Biochim Biophys Acta 2015, 1852(5):882-892.

33. Menzies FM, Garcia-Arencibia M, Imarisio S, O'Sullivan NC, Ricketts T, Kent BA, Rao MV, Lam W, Green-Thompson ZW, Nixon RA et al: Calpain inhibition mediates autophagy-dependent protection against polyglutamine toxicity. Cell Death Differ 2015, 22(3):433-444.

\section{Figures}




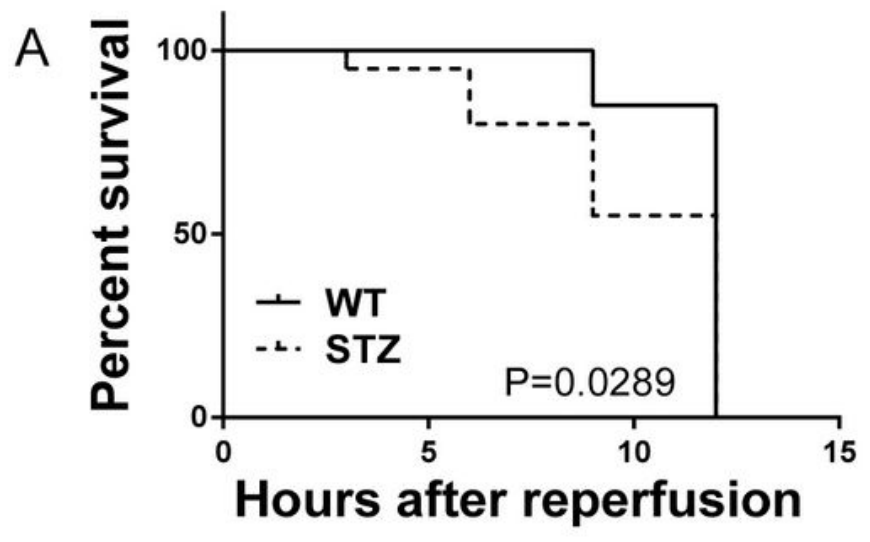

B

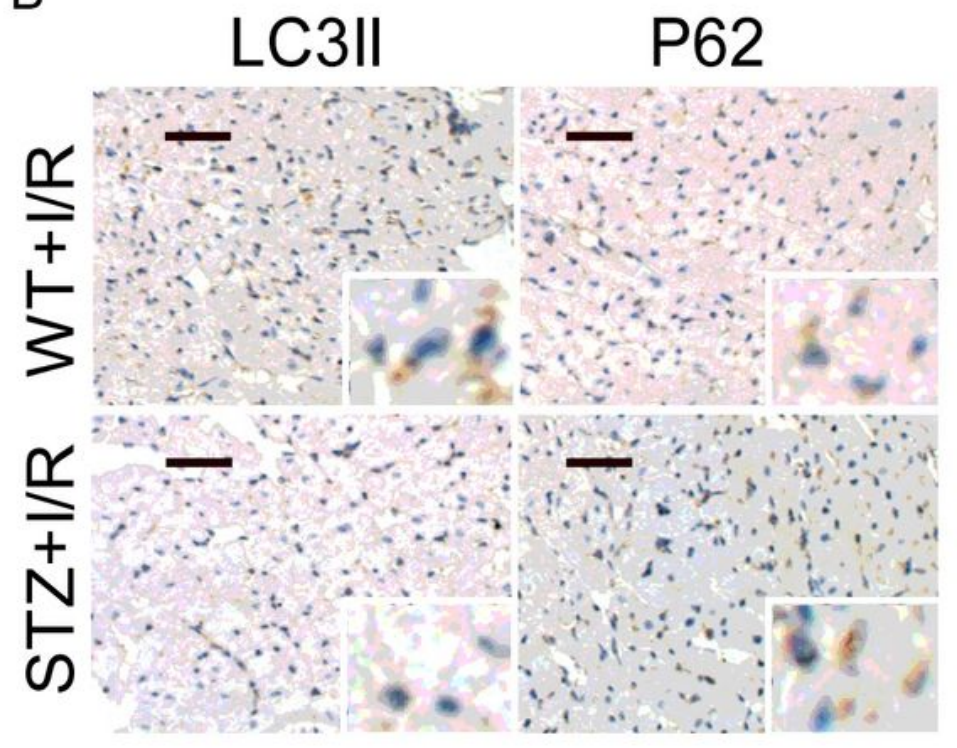

D

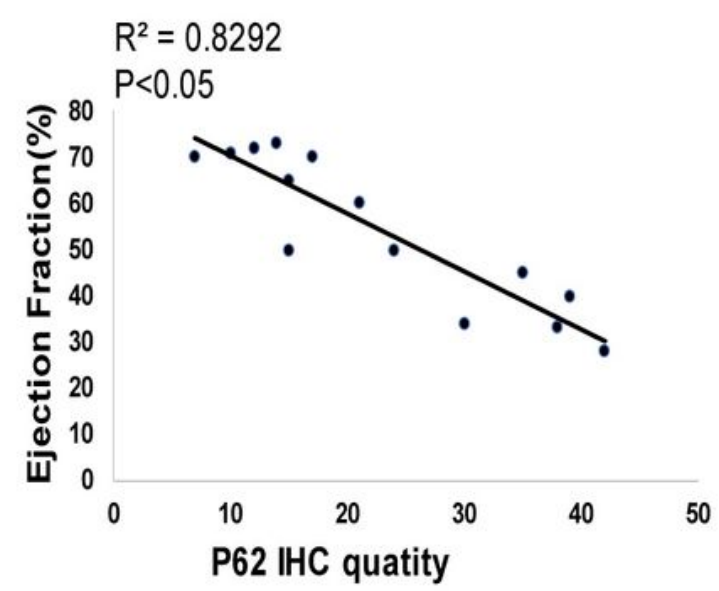

C

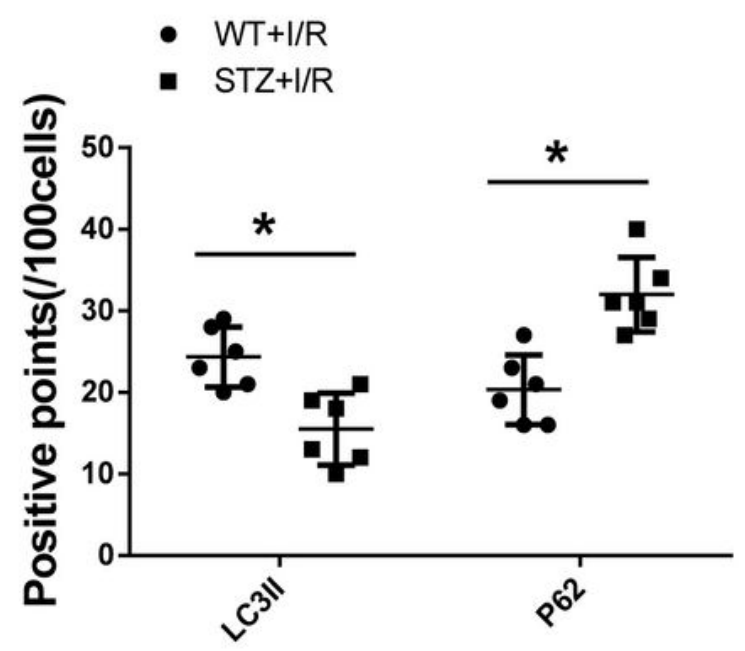

\section{Figure 1}

Autophagic flux correlates with I/R injury of STZ-induced diabetic mice. (A) Survival curve analysis of the WT mice $(n=12)$ and the diabetic mice $(n=12)$. $(B, C)$ immunohistochemical staining of P62 and LC3II in WT and diabetic heart samples taken after $12 \mathrm{~h}$ of reperfusion. Quantification of the positive staining level is shown (dots/100 cells). The magnification is 100 and 400 times. (D) Linear correlation between P62 expression and EF value. * $p<0.05$ 

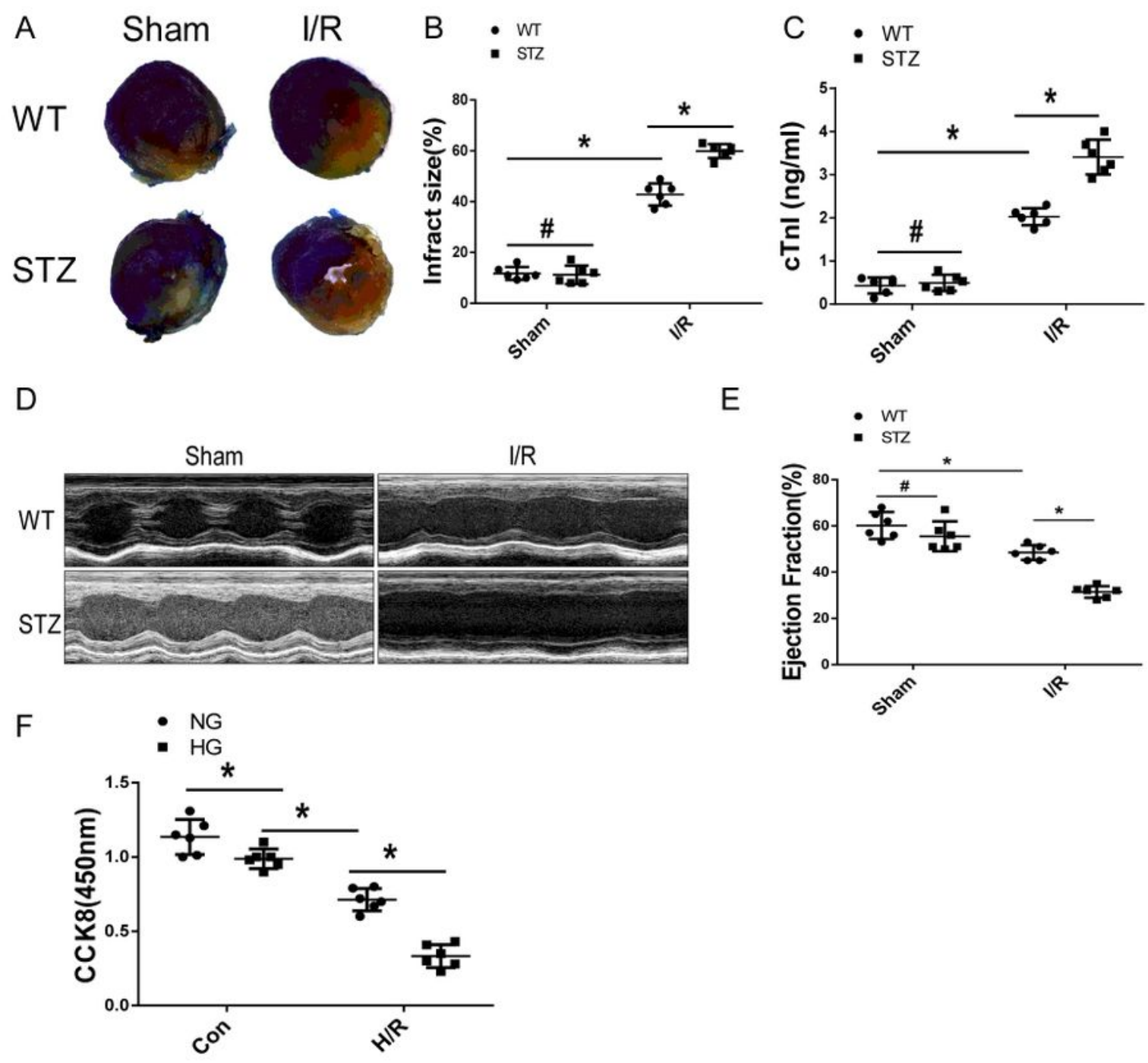

E
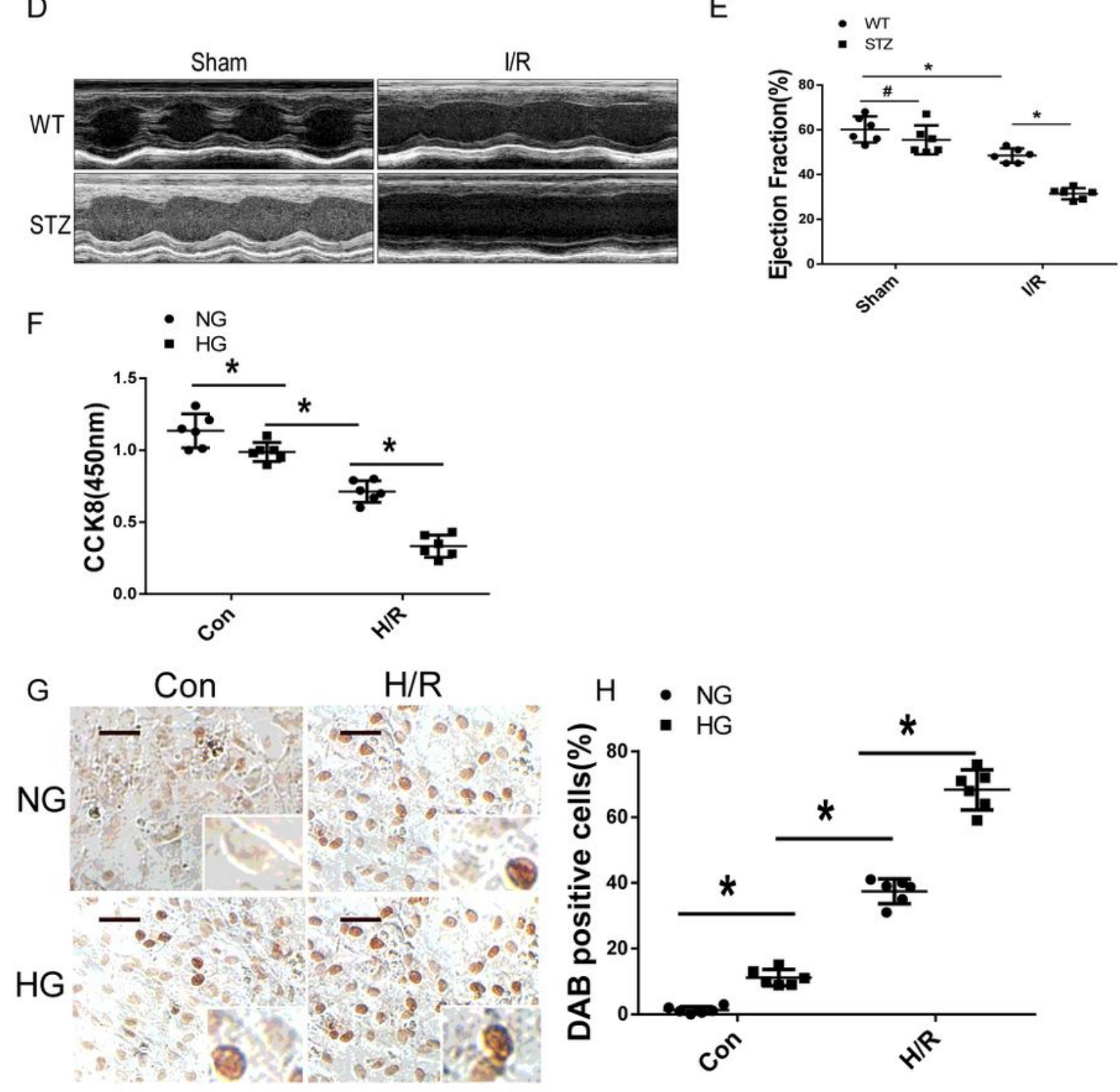

Figure 2

Diabetic mice are more sensitive to I/R injury, in vivo and in vitro. (A, B) WT and diabetic mice submitted to $\mathrm{I} / \mathrm{R}$ or sham operated. Evans blue-TTC staining showed the myocardial infarction size, and quantitative analyses. (C) Serum cTnl at $12 \mathrm{~h}$ of reperfusion by ELISA. (D, E) Cardiac function (EF) by echocardiography. (F) Cell viability by CCK8 assay. (G, H) Apoptosis in cultures cardiomyocytes by TUNEL, and quantitative analyses. * $p<0.05 ; \# p>0.05$ ( $n=6$ per group). 
A
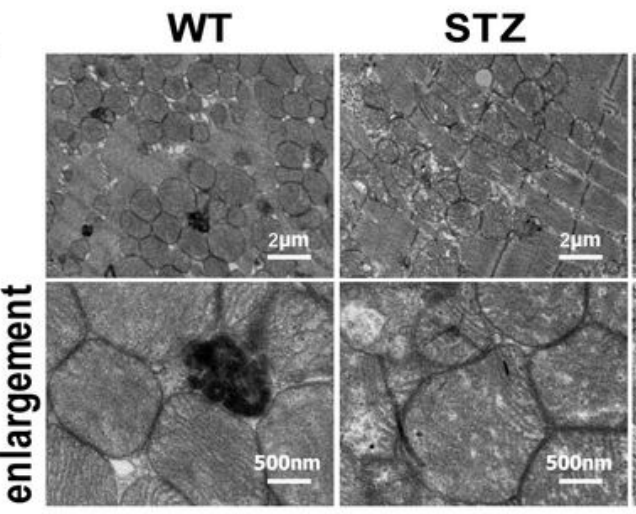

I/R

I/R+STZ
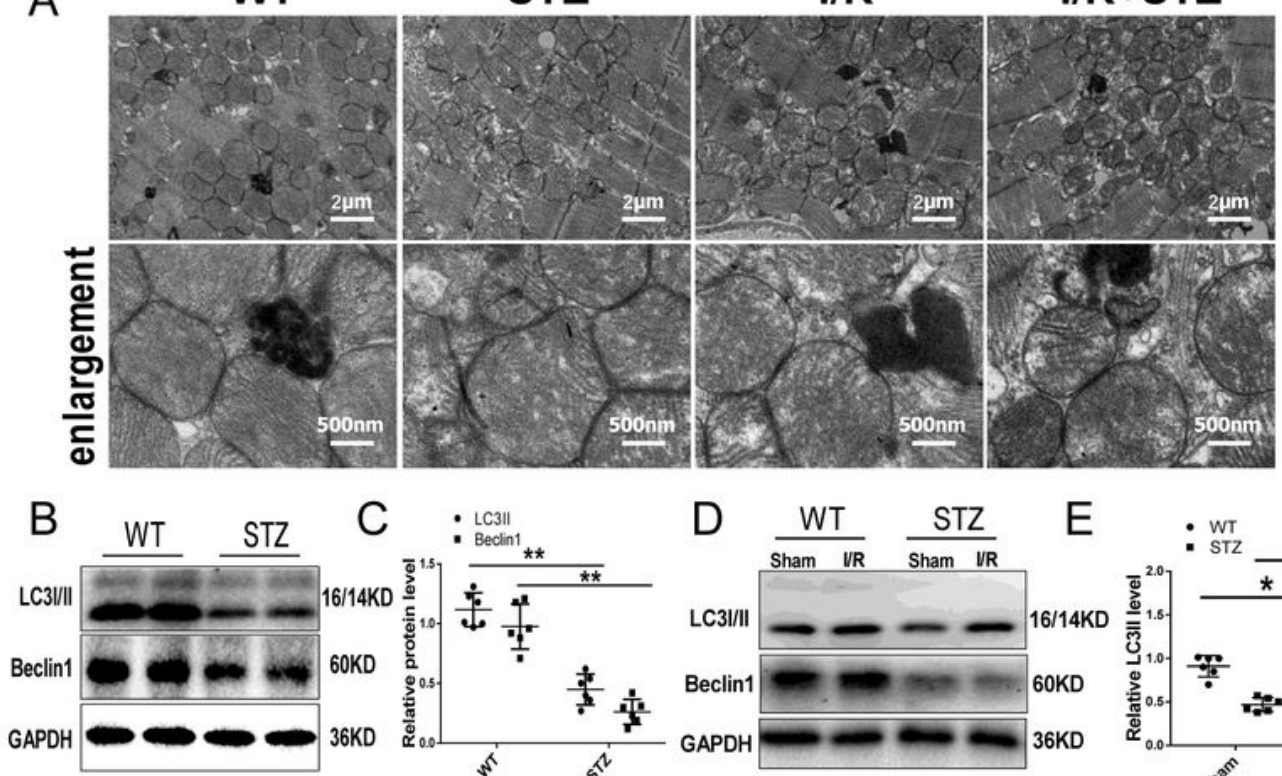

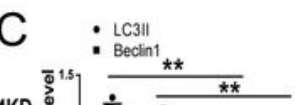

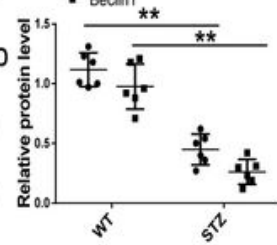

D $\frac{\text { WT }}{\text { Sham UR }} \frac{S T Z}{\text { Sham VR }}$
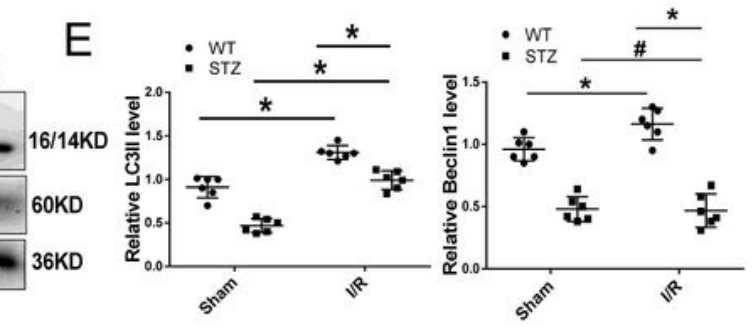

$\mathrm{F}$
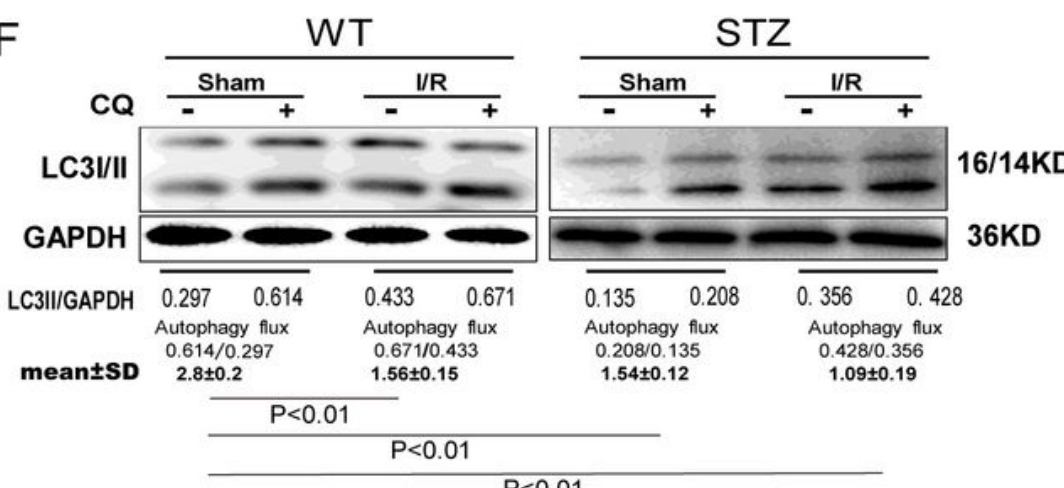

$1.54 \pm 0.12$

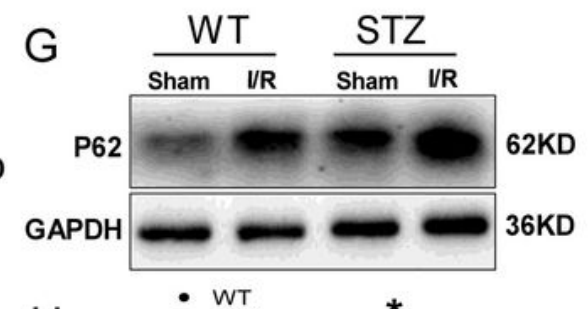

$\mathrm{H}$
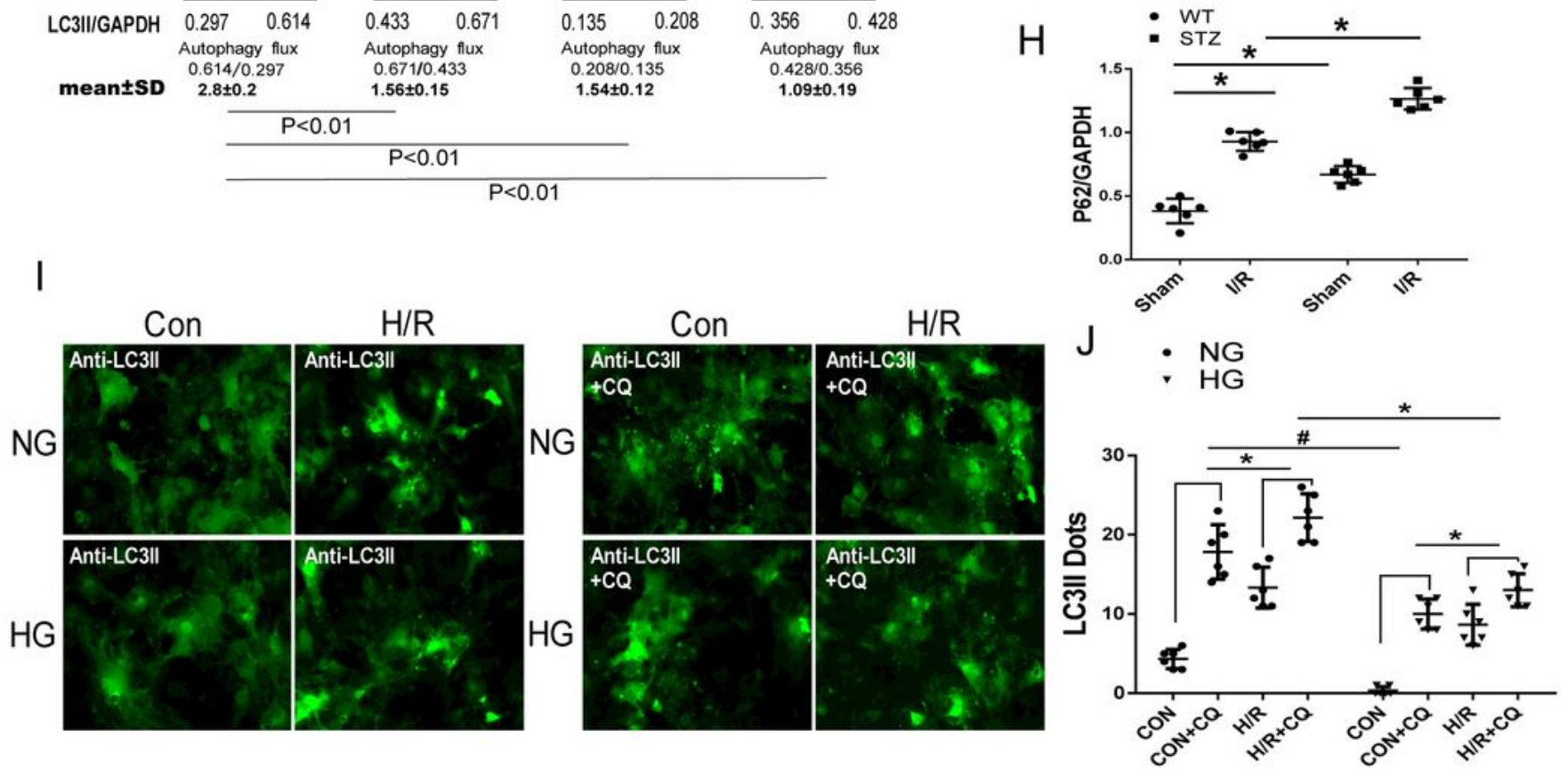

\section{Figure 3}

Autophagic flux was Impaired in diabetic IR injury. (A) Representative illustrations of TEM showed autophagosome and mitochondrial ultrastructural and in each group. (B-E) Western blot and quantitative analyses of LC3II and Beclin1 ( $n=6$ per group). (F) Autophagic flux were accessed through testing LC3 expression by western blot with $C Q$ intervention in diabetic l/R mice. $(G, H)$ P62 expression by western blot and quantitative analyses. (I) Autophagic flux were detected through testing LC3 expression with CQ 
intervention by immunofluorescence in cultured cardiomyocytes. (magnification x200). (J) In the field of view of the same size, we compared the difference between the group with CQ and the group without CQ. * $p<0.05 ; \# p>0.05$. ( $n=6$ per group)

A
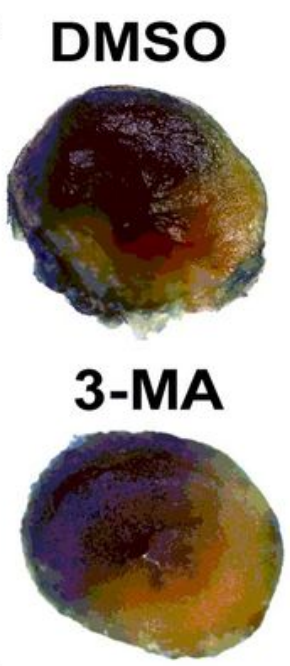

C

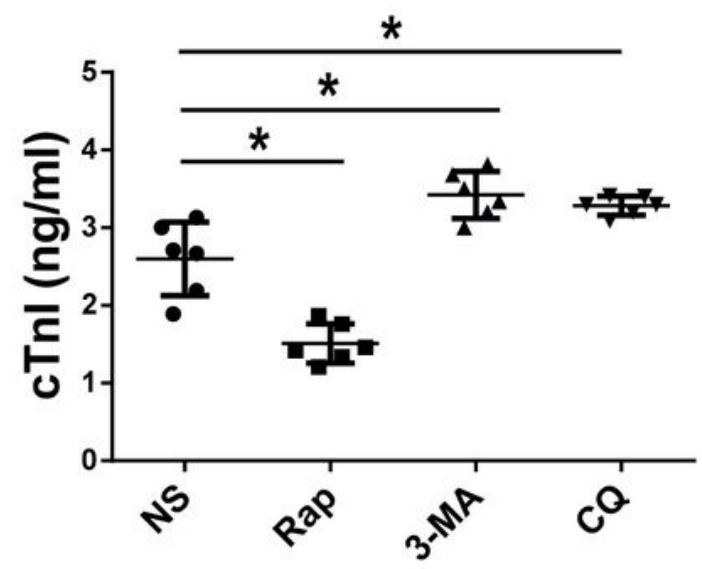

D

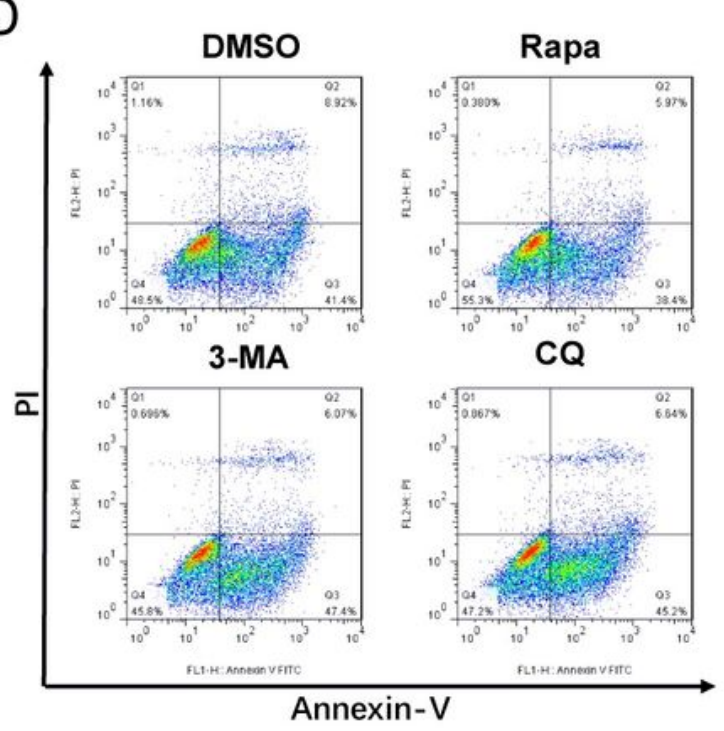

B

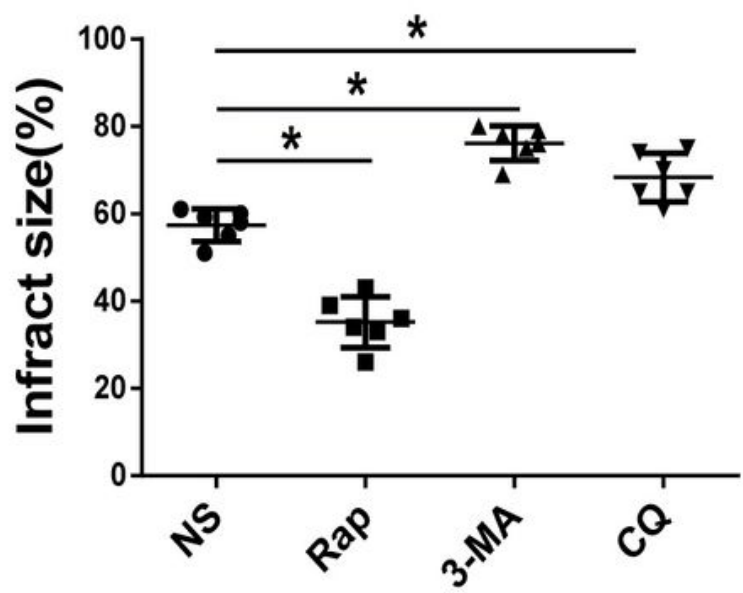

$\mathrm{E}$

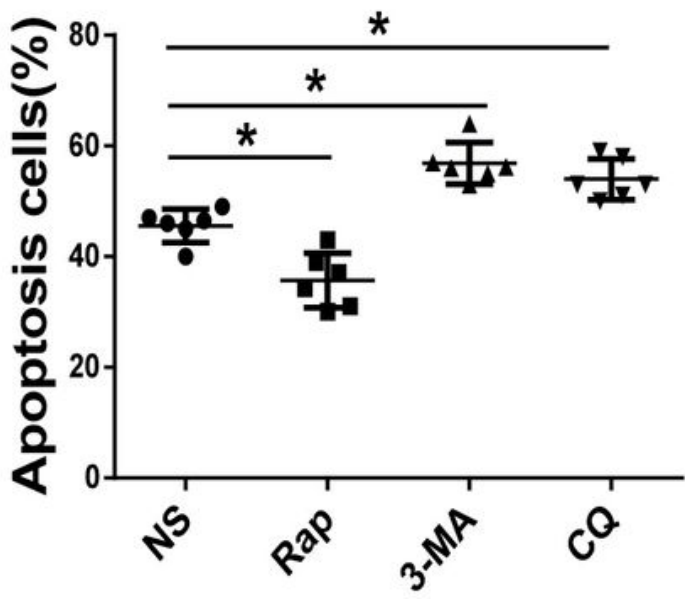

Figure 4

Regulating autophagic flux impact I/R injury in the diabetic heart. (A, B) Myocardial infarction size by Evans blue-TTC staining of diabetic mice subjected to I/R after rapamycin $(1 \mathrm{mg} / \mathrm{kg}), 3-\mathrm{MA}(30 \mathrm{mg} / \mathrm{kg})$ or 
CQ (60 mg/kg) intervention, and quantitative analyses. ( $\mathrm{n}=6$ per group), (C) Serum cTnl level by ELISA. (D, E) Apoptosis by flow cytometry of cultured cardiomyocytes underwent $\mathrm{HG}$ and $\mathrm{H} / \mathrm{R}$ and pretreated with DMSO, rapamycin $(0.2 \mu \mathrm{M})$, 3-MA $(5 \mathrm{mM})$ or CQ $(10 \mu \mathrm{M})$, and quantitative analyses * $p<0.05$.

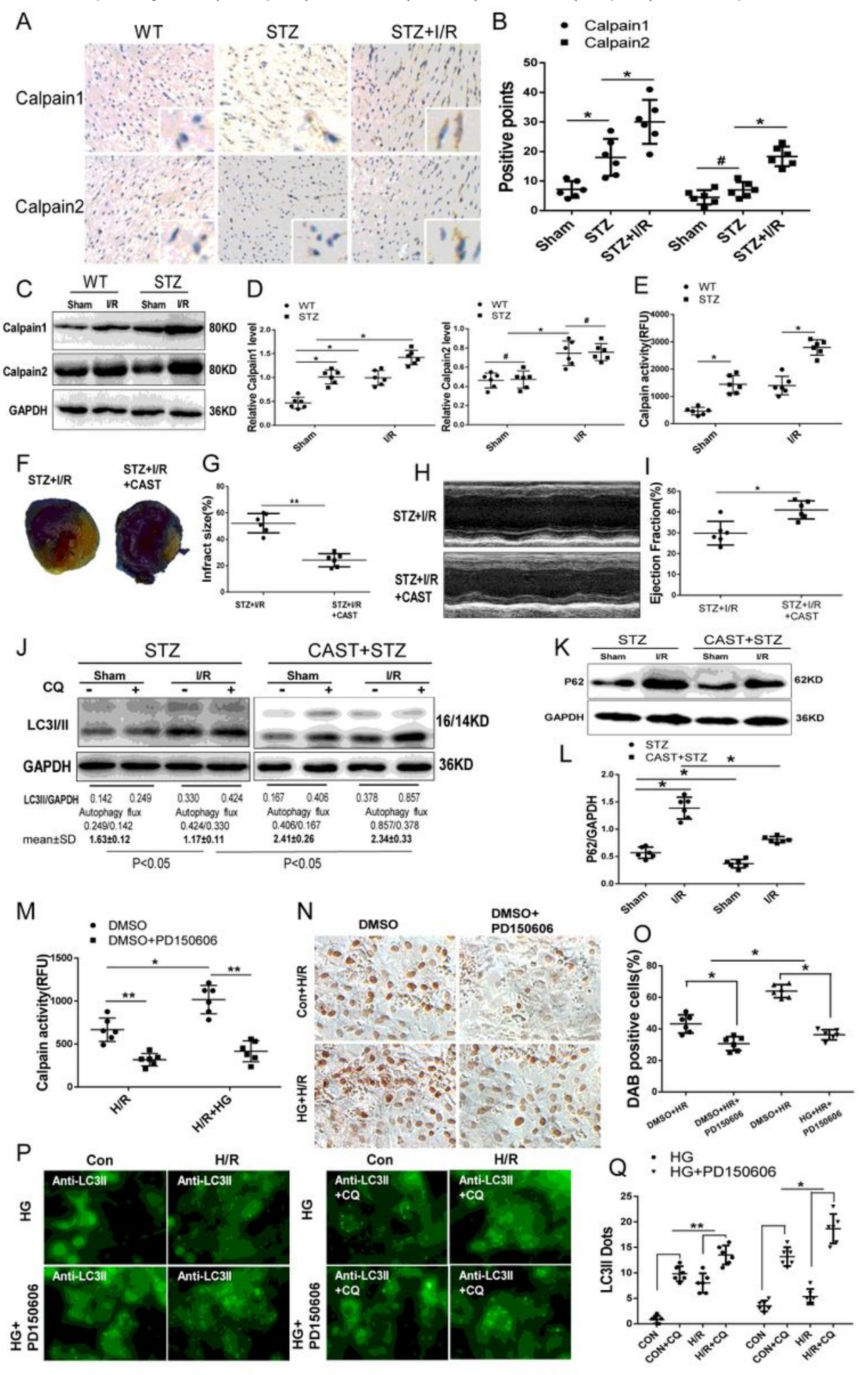

Figure 5

Calpains are activated and aggravates I/R injury in diabetic heart. (A-D) Cardiac calpain1 and calpain2 expression by IHC and western blotting, and quantitative analyses. (E) Calpain activity by N-succinyl- 
LLVY-AMC fluorometry. (F, G) Myocardial infarction size Evans blue-TTC staining in WT mice and TgCAST mice with diabetic I/R, and quantitative analyses. $(\mathrm{H}, \mathrm{I})$ Cardiac function (EF) by echocardiography in WT mice and Tg-CAST mice with diabetic I/R, and quantitative analyses. ( $\mathrm{J}$ ) Autophagic flux by western blot in each group in WT mice and Tg-CAST mice with diabetic I/R. (K, L) P62 expression by western blot. (M) Calpain activity of cultured cardiomyocytes in $H / R$ and $H / R+H G$ treatment cardiomyocytes. $(\mathrm{N}, \mathrm{O})$ Apoptosis of cultured cardiomyocytes by TUNEL. (P, Q) Autophagic flux (LC3 expression under CQ intervention) by immunofluorescence (magnification $x 200)$. ${ }^{*} p<0.05$; ${ }^{\star *} p<0.01$; \# $p>0.05$. ( $n=6$ per group). 

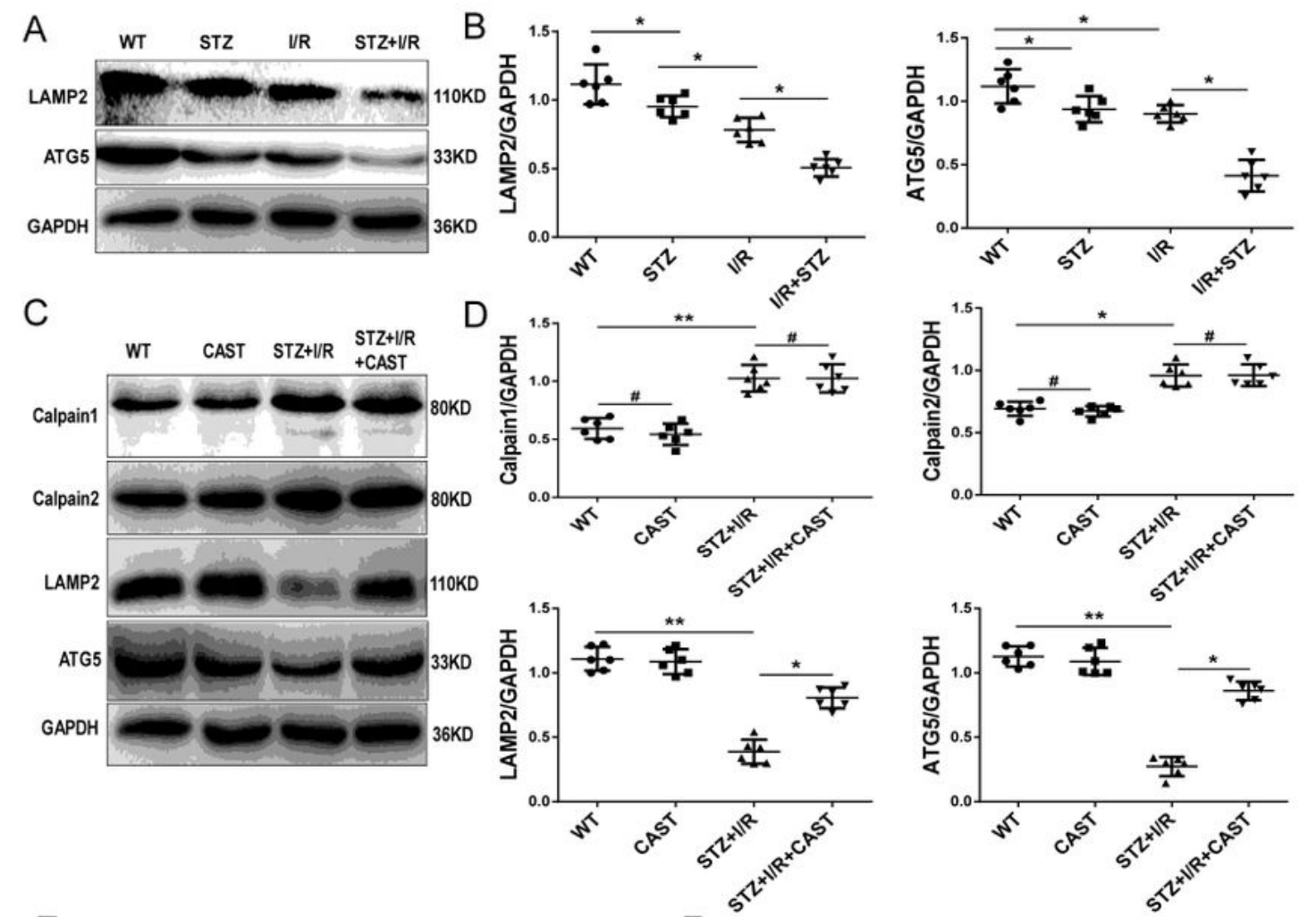

$\mathrm{E}$

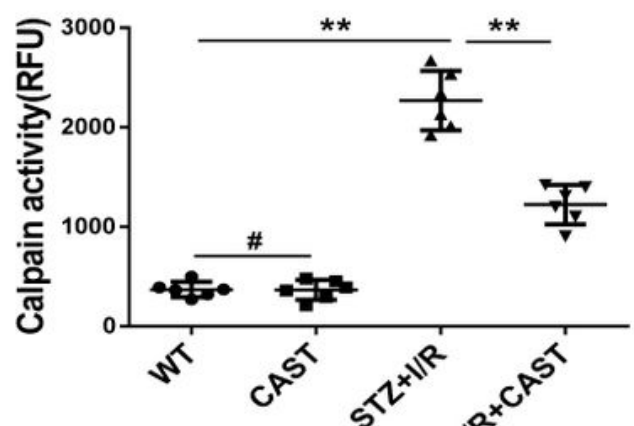

$\mathrm{F}$
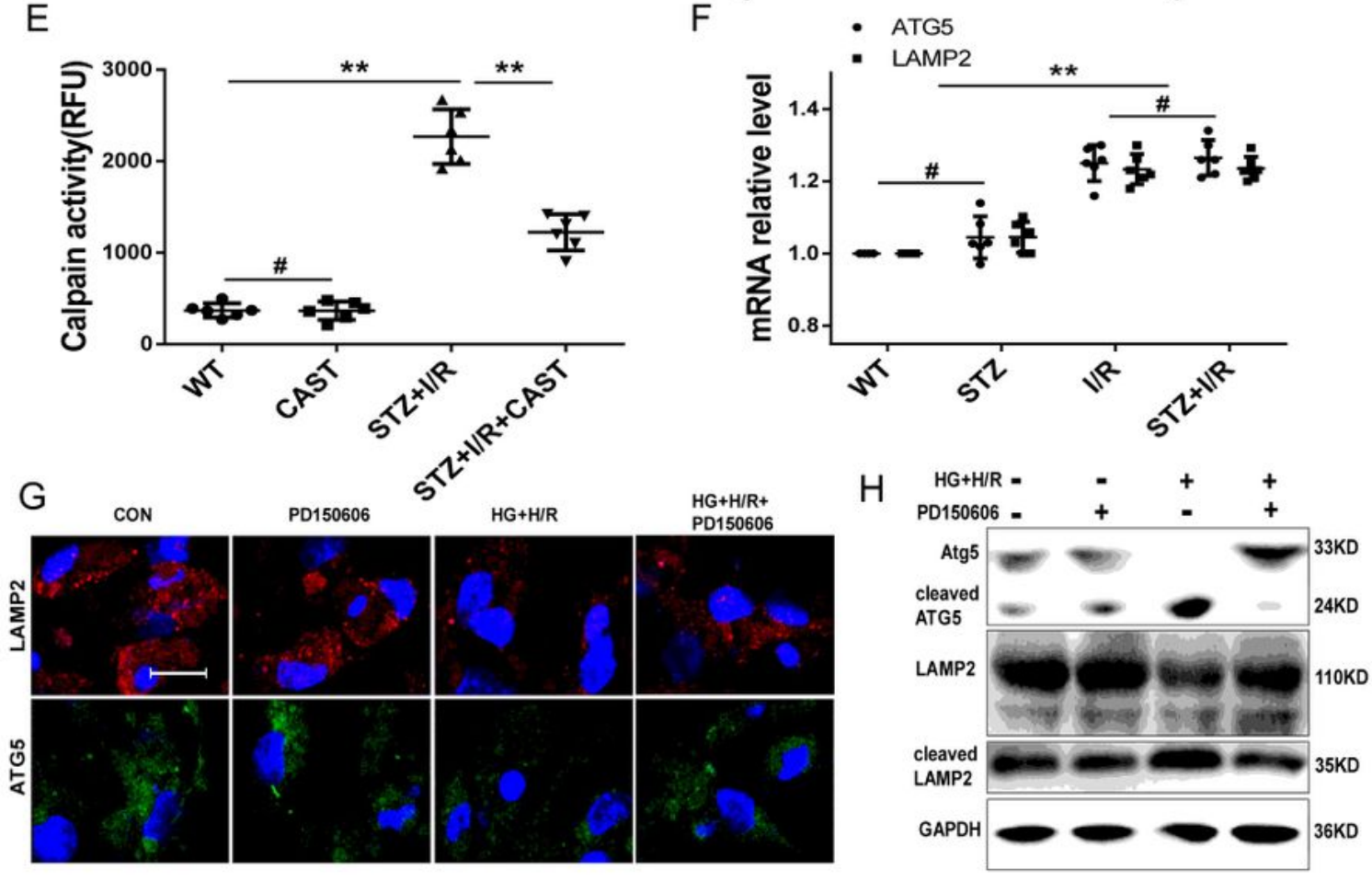

\section{Figure 6}

Calpains degrades ATG5 and LAMP2 during diabetic l/R. (A, B) The expression LAMP2 and ATG5 by western blotting, and quantitative analyses. ( $n=6$ per group). (C, D) Calpain1, calpain2, LAMP2 and ATG5 expression by western blotting, and quantitative analyses. $(n=6$,$) . (E) Calpain activity by fluorometry.$ $(n=6)$. (F) ATG5 and LAMP2 mRNAs level by RT-qPCR. $(n=6)$. (G) LAMP2 (red) and ATG5 (green) 
expression by immunofluorescence (bar=20 $\mu \mathrm{m})$. $(\mathrm{H})$ The cleaved fragment of LAMP2 and ATG5 by western blotting. * $p<0.05 ; * \star ~ p<0.01 ; \# p>0.05$. ( $n=6$ per group).
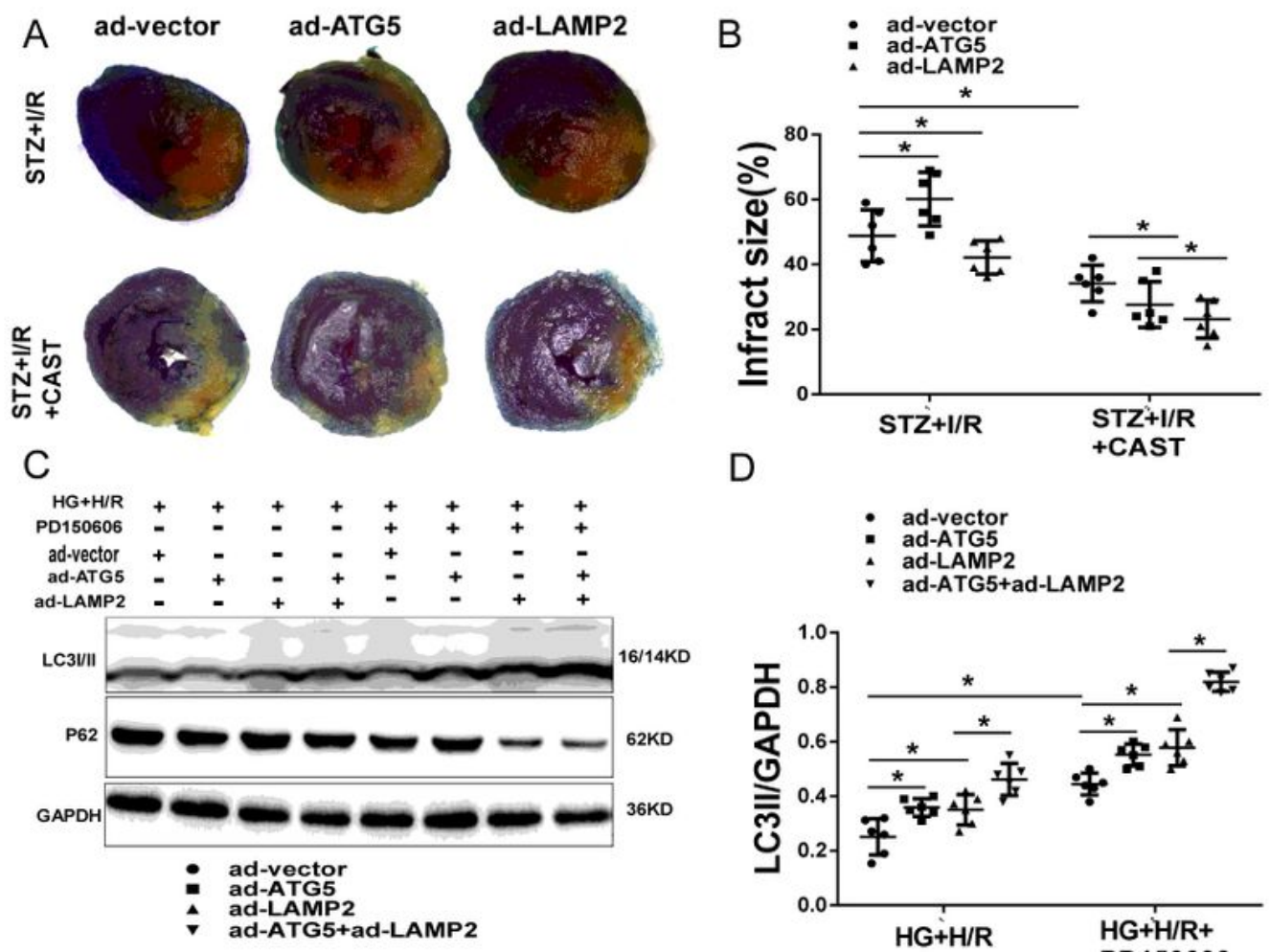

D
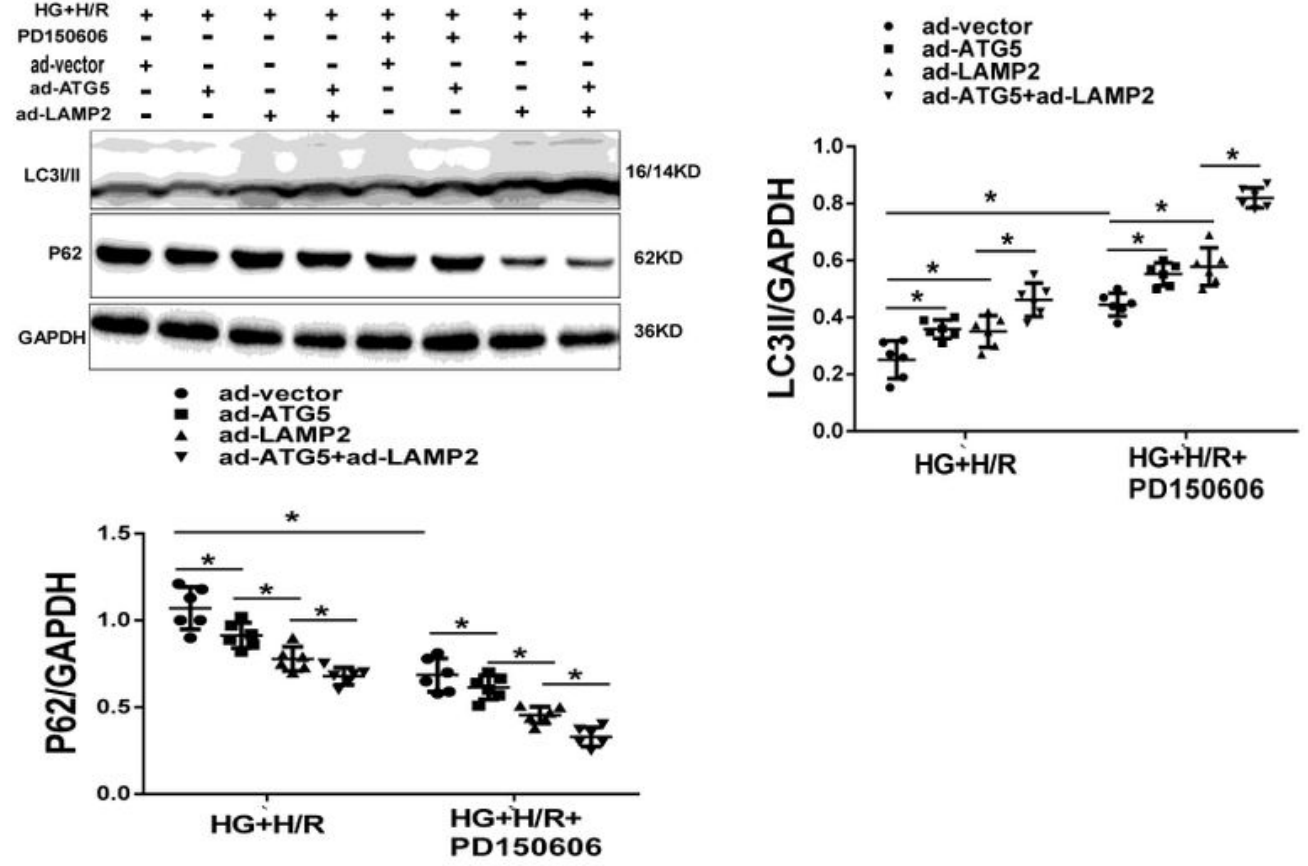

E

- ad-vector

ad-ATG5

- ad-ATG5+ad-LMPA2
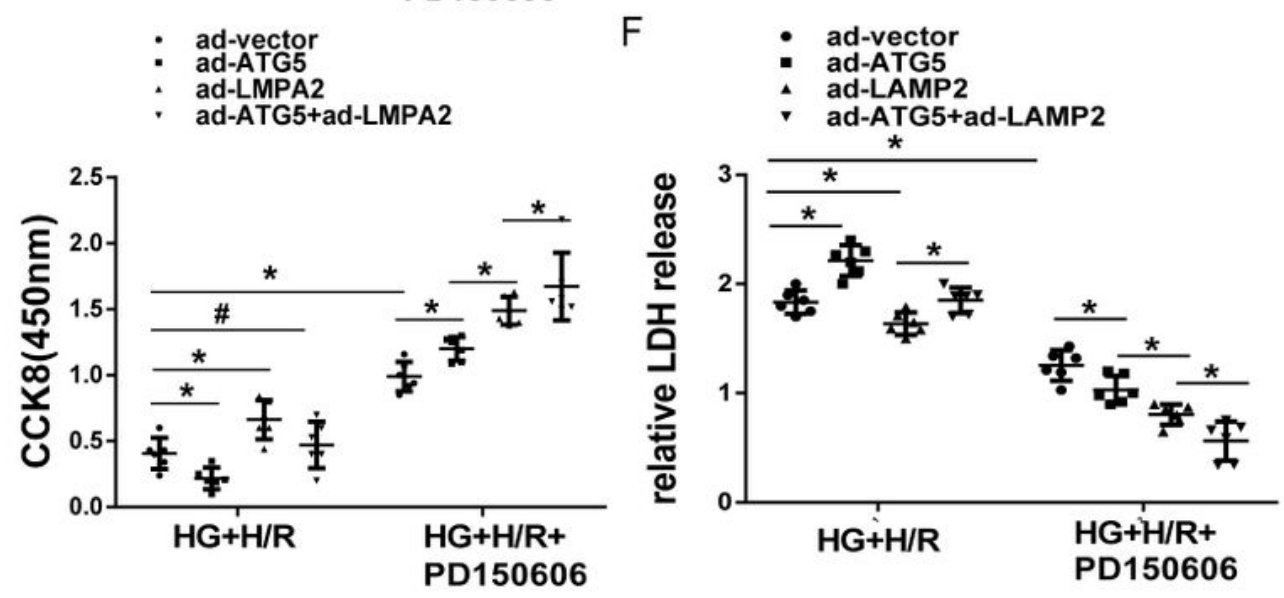

Figure 7

Effect of overexpression of ATG5 or LAMP2 on cardiomyocytes with diabetic I/R injury. (A) Diabetic WT and Tg-CAST mice were transfected with ad-vector, ad-ATG5 or ad-LAMP2 for 3 days, and then subjected to I/R. Myocardial infarction size by Evans blue-TTC staining, and quantitative analyses. (C, D) Cultured 
cardiomyocytes were transfected with ad-GFP, ad-ATG5 or ad-LAMP2 for $72 \mathrm{~h}$ and then subjected to H/R. ATG5 and LAMP2 expression by western blotting, and quantitative analyses. (E) P62 expression of cultures cardiomyocytes by western blotting, and quantitative analyses. (F) Cardiomyocytic viability by CCK8. (G) LDH concentrate of cell culture medium by LDH assay. ${ }^{*} p<0.05 ; \# p>0.05$. ( $n=6$ per group).

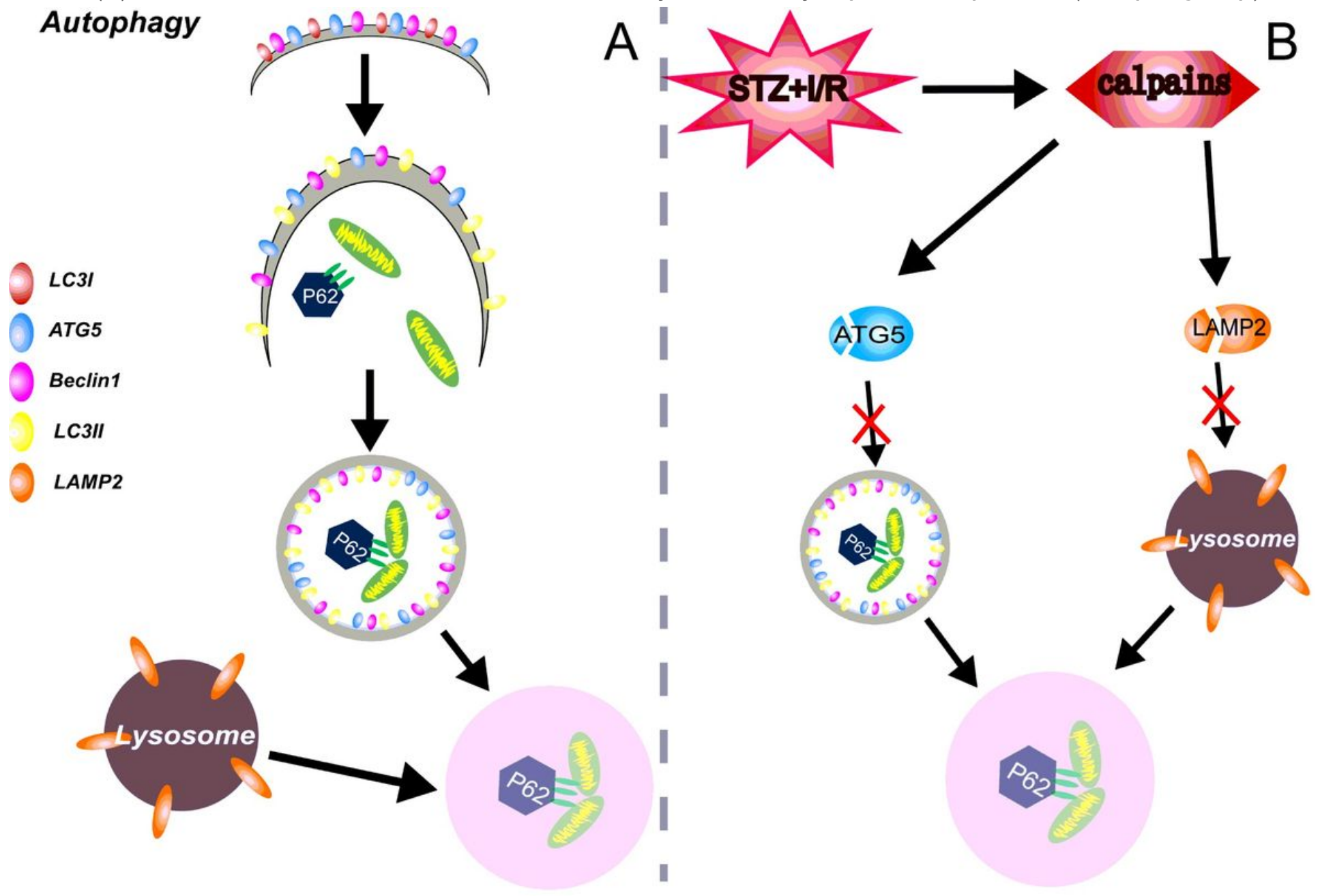

Figure 8

Figure 8

\section{Supplementary Files}

This is a list of supplementary files associated with this preprint. Click to download.

- supplymentary1.tif 